\title{
Pneumocephalus as a rare complication: a systematic review plus clinical vignette
}

\author{
Katarzyna Śmiłowska ${ }^{1^{*}}$, Katarzyna Sznajder-Stacha ${ }^{2^{*}}$, Daniel Kocyłowski ${ }^{3}$, Aleksandra Popek ${ }^{4}$, \\ Kamila Rozpondek ${ }^{2}$, Maciej Grechuta ${ }^{2}$, Mehri Salari ${ }^{5}$ \\ ${ }^{1}$ Department of Neurology, Regional Specialist Hospital im. Św. Barbary in Sosnowiec, Poland \\ ${ }^{2}$ Department of Neurology, City Hospital in Sosnowiec, Poland \\ ${ }^{3}$ Department of Neurosurgery, School of Medicine in Katowice, Medical University of Silesia, Katowice, Poland \\ ${ }^{4}$ Department of Radiology, City Hospital in Sosnowiec, Poland \\ ${ }^{5}$ Functional Neurosurgery Research Centre, Shohada Tajrish Neurosurgical Centre of Excellence, Shahid Beheshti University \\ of Medical Sciences, Tehran, Iran
}

\begin{abstract}
Introduction. Pneumocephalus is a clinical entity characterised by the presence of gas in the intracranial space. It can result from many different causes. The most common cause is head or facial trauma. Other causes include neoplasms, infections, and surgical or diagnostic procedures. Spontaneous non-traumatic pneumocephalus is a rare condition caused by bone defects, malformations, infections, tumours, intravenous air injection, and other causes. This review, supplemented with a case presentation, aims to summarise the current state of knowledge regarding non-traumatic pneumocephalus.

Methodology. This review involved an electronic search (PubMed, Scopus, Embase, and Web of Science) to identify studies regarding non-traumatic pneumocephalus. In addition, reference lists of identified articles were screened for other potentially relevant papers.

Results. In total, 1,107 articles were retrieved by searching databases with the selected query. Based on the selection process, 134 articles were included. These articles were then classified into 'otogenic,' 'bone defect','malformations', 'infectious,' tumours', 'associated with intravenous air injection', and other categories.

Conclusion. Spontaneous non-traumatic pneumocephalus is a rare condition. Symptoms, clinical courses, and prognoses vary depending on the underlying cause of the disease. To the best of our knowledge, this review's example is the first case report of spontaneous pneumocephalus due to air embolism secondary to lung cancer.
\end{abstract}

Key words: pneumocephalus, spontaneous, non-traumatic, atraumatic

(Neurol Neurochir Pol 2021; 55 (3): 253-268)

\section{Introduction}

Pneumocephalus is characterised by the pathological collection of gas within the cranial cavity [1]. Pneumocephalus was first described in 1741 by Lecat et al. [2]. The main cause of pneumocephalus is head trauma, which is responsible for up to $74 \%$ of all cases. Other causes of pneumocephalus include: (1) intracranial neoplasms; (2) infections; (3) neurosurgery; (4) paranasal sinus surgery; and (5) diagnostic or neurosurgical interventions such as pneumoencephalography or lumbar puncture [3].

However, in rare cases, pneumocephalus can occur spontaneously. Spontaneous occurrence represents only $0.6 \%$ of all

Address for correspondence: Katarzyna Śmiłowska, Sosnowiecki Szpital Miejski, Oddział Neurologii, Szpitalna 1 Str., 41-219 Sosnowiec, Poland, e-mail: kasia.smilowska@gmail.com

* These authors are to be considered as joint first authors 
cases and was first described in 1954 by Jelsma and Moore [4]. Based on anatomical localisation, pneumocephalus is classified as 'extradural', 'subdural', 'subarachnoidal,' 'intraparenchymal' or 'intraventricular'. Two factors contribute to the pathomechanism of pneumocephalus: a decrease in intracranial pressure and coexisting defects in the dura. These factors are caused either by a ball-valve mechanism which causes a rapid increase in the volume of air inside the skull cavity without compensatory cerebrospinal fluid (CSF) outflow, or by CSF leakage which creates intracranial hypotension resulting in an aspiration of air $[3,5,6]$.

Spontaneous non-traumatic pneumocephalus is a rare condition. Few case studies have been published to date [5]. The most common causes include (1) otogenic pneumocephalus, (2) bone defects, (3) malformations (birth defects), (4) infections, (5) tumours, (6) other causes, and (7) an intravenous injection of air. Here, we present the case of a patient with non-traumatic pneumocephalus, and provide a literature review on other non-traumatic cases.

\section{Clinical vignette}

\section{Patient 1}

A 66-year-old right-handed man with a past medical history of lung cancer was found at home, unconscious. His family reported a persistent hiccup, which had occurred the day before admission. Upon neurological examination, the patient was unconscious. He was intubated due to respiratory insufficiency, and his pupils were small, with anisocoria $\mathrm{R}>\mathrm{L}$ and a poor response to light. He did not respond to pain stimuli. Computer tomography (CT) of the patient's brain showed a massive cerebral air embolism, which was later classified as an example of intra-axial intravascular pneumocephalus (Fig. 1). A thoracic CT scan revealed fluid and solid components of the left hilus with an obstruction of the left main bronchi (Fig. 2). After contrast enhancement, clear infiltration was observed, including the left pulmonary veins, left atrium, and left ventricle. An intra-infiltrating mass included lucencies, indicating the presence of gas or air. Left cardiac lung cancer infiltration with airway communication was strongly suspected. The patient died a few hours after his admission to the emergency department.

\section{Methodology}

The references used in the current review were primarily identified by performing a systematic search of the PubMed and Web of Science databases. The search queries used included 'pneumocephalus', 'spontaneous', 'non-traumatic', 'atraumatic', and 'cancer'. The title/abstract filter was used to broaden the search. The final search was performed in April 2020. In addition, reference lists of identified articles were screened for other potentially relevant papers. Articles were selected based on eligibility criteria. Studies were included if

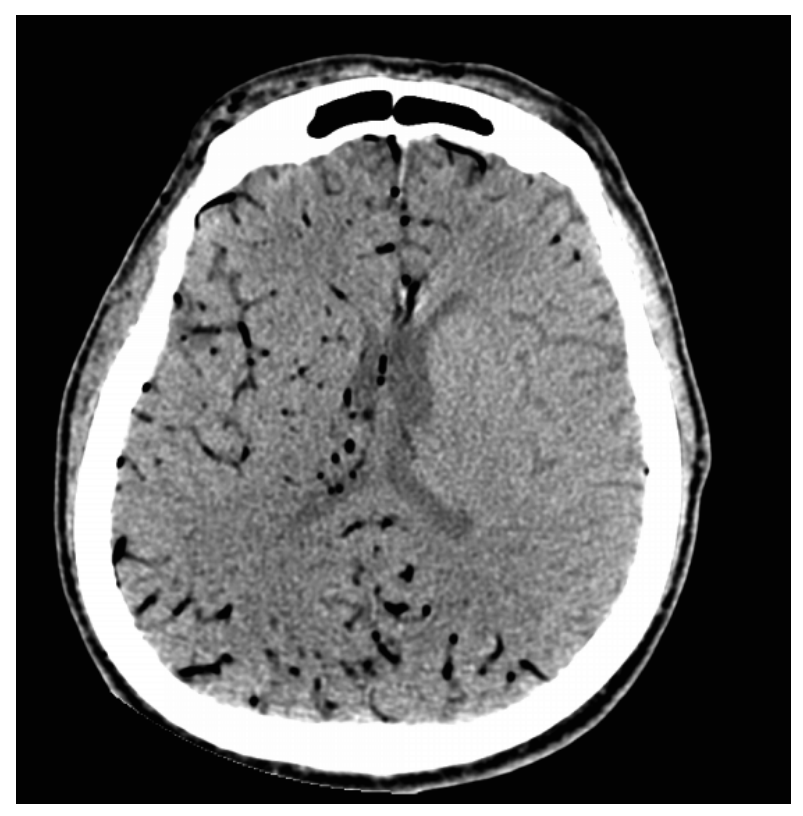

Figure 1. Brain CT. On axial CT, scan shows brain vessels with low density that indicates intravascular pneumocephalus

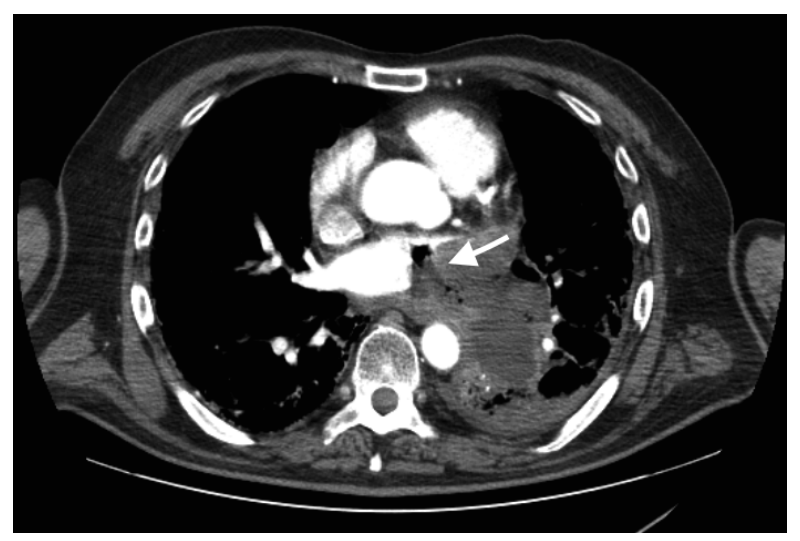

Figure 2. Chest CT. Axial CT-scan. Arrow indicates neoplasmic lung cancer and pulmonary vein infiltration with cancer mass, resulting in bronchial-vein fistula

(1) they presented original research, (2) they were conducted on pneumocephalus, and (3) they were written in English.

\section{Selection process}

In total, 1,107 articles were retrieved from the database search using the chosen queries (Fig. 3). Based on the selection process, 134 articles were included. These articles were then categorised as follows: (1) 'otogenic' (44 studies), (2) 'bone defect' (10 studies), (3) 'malformations' (five studies), (4) 'infectious' (38 studies), (5) tumours (30 studies), (6) others (four studies), and (7) associated with intravenous air injection (three studies). 


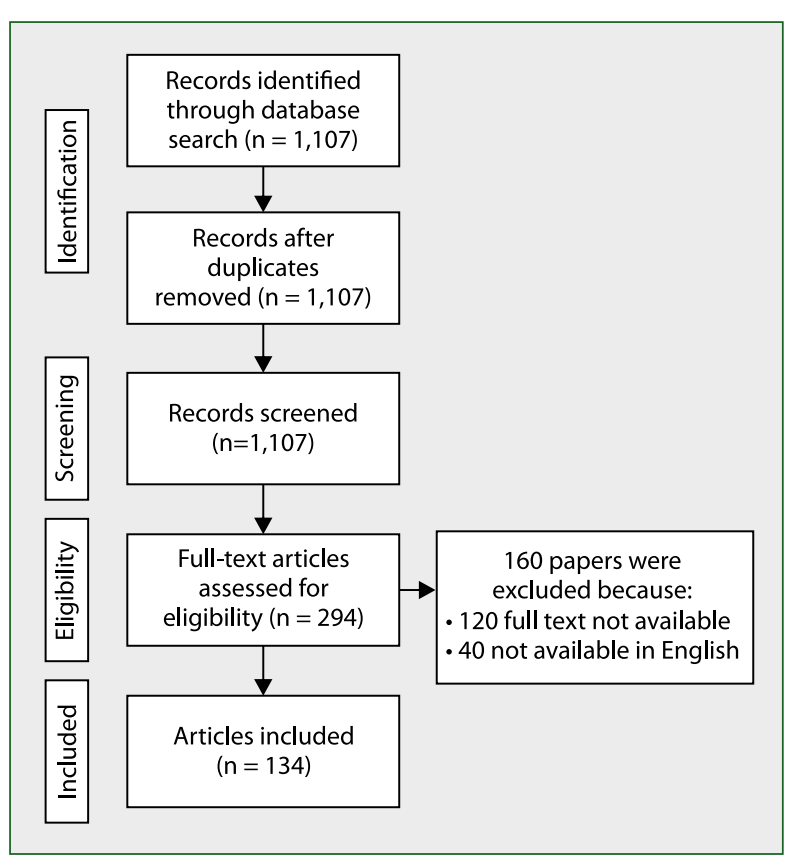

Figure 3. Selection process

\section{Results}

Spontaneous otogenic pneumocephalus (SOP)

A total of 44 articles were retrieved from the databases (Table 1). These articles were case studies reporting interesting patients and mechanisms of spontaneous otogenic pneumocephalus (SOP). The most common cause of SOP is a congenital bone defect in the pneumatised cells surrounding the middle ear. This defect develops into a fistulous communication with the intracranial compartment. Various factors can contribute to this mechanism, including: the Valsalva manoeuvre (coughing, sneezing, nose blowing, exhaling), the Politzer manoeuvre (blowing air through the nose during swallowing), and exposure to significant pressure changes (e.g. air travel) [7-11]. However, in a significant number of cases, the trigger remains unknown [12]. Wilkinson et al. reported a patient with intraventricular SOP related to barotrauma, which was due to tegmen defects that were confirmed in neuroimaging studies [13]. As such, bone defects in the middle cranial fossa have been proven to be associated with a risk of SOP occurrence [13]. Additionally, in SOP, hyperpneumatisation mastoids are a relatively common phenomenon [14]. Dowd et al. claimed that both a defect in the bones surrounding the middle ear (e.g. tegmen tympani defect) and a pressure difference on both sides of the defect are necessary for the formation of otogenic pneumocephalus [12]. Other descriptions of SOP in the literature support this theory $[9,10,15]$. Headache is the most common symptom of SOP, followed by aphasia $[12,16]$. Villa et al. and Krayenbühl et al. reported a similar case of intraparenchymal pneumocephalus which manifested clinically with abnormal acoustic sensations, aphasia, and visual-field disturbances
$[16,17]$. The symptoms often described in the literature also include tinnitus, fullness in the ear, rhinorrhoea, nausea, and confusion $[11,13,18,19]$.

\section{Pneumocephalus associated with bone defects}

A total of 10 articles were retrieved from the databases (Tab. 1). These articles contained case reports of pneumocephalus resulting from bone defects other than those related to SOP. In this group, the most frequent were defects of sphenoid sinus and cribriform plate causing hyperpneumatisation of the cranium $[20,21]$. This process leads to communication between the inside of the skull and the intracranial compartment, which allows air to penetrate the cranium. Symptoms may be preceded by the Valsalva manoeuvre (e.g. blowing the nose, sneezing) [22,23], though in a significant number of cases the triggers remain unknown [24]. Similarly to SOP, headache is the most commonly described symptom, along with rhinorrhoea $[20,24]$. Nash et al. presented the case of a 27 -year-old woman diagnosed with intraparenchymal pneumocephalus which manifested with a sudden onset of alien limb syndrome [9]. This rare phenomenon is most commonly described in cortico-basal syndrome and associated with frontal lobe and corpus callosum changes. For the reported patient, alien limb syndrome was related to pneumocephalus localised in the right frontal lobe, due to a cribriform plate defect. After treatment (pneumocephalus aspiration and bone defect repair), the patient recovered completely. Tension pneumocephalus is a relatively rare condition: only two articles contain case reports of tension pneumocephalus associated with bone defects $[20,25]$.

\section{Pneumocephalus associated with malformations}

A thorough search of the selected databases yielded a total of five articles on pneumocephalus associated with malformations. In all of these cases, pneumocephalus was associated with malformations related to an open myelomeningocele with accompanying CSF leakage [26]. In three of the five cases, pneumocephalus was localised intraventricularly [26-28]. The other locations were the posterior fossa and infratentorial area $[29,30]$. Spontaneous pneumocephalus occurs in a mechanism called the 'reverse bottle effect', which is attributed to CSF leakage through the neural tube defect. The air passes into the subarachnoid space and then through the spinal canal into the cranial cavity. In the case of open myelomeningocele, the air enters the spinal canal (e.g. when a child cries), which is associated with an increase of intracranial pressure accompanied by an outflow of CSF, leading to the formation of negative pressure in the ventricular system and an aspiration of air from outside $[26,28]$. The most commonly described symptoms are breathing difficulties and paresis of the lower limbs $[27,28]$.

\section{Pneumocephalus associated with infections}

A total of 37 articles on infections and pneumocephalus were retrieved from the databases (Table 1). Infections are a relatively common cause of pneumocephalus, and they 
Table 1. Non-traumatic pneumocephalus: studies included in review

\begin{tabular}{|c|c|c|c|c|c|}
\hline \multicolumn{6}{|c|}{ Spontaneous otogenic pneumocephalus } \\
\hline Authors [year] & Aetiology & Location & Symptoms & $\begin{array}{l}\text { Location } \\
\text { of air entry }\end{array}$ & Other \\
\hline Barry [2019] [57] & Spontaneous & Posterior cranial fossa & $\begin{array}{l}\text { Confusion, lethargy, myalgia, } \\
\text { cough, fever }\end{array}$ & Mastoid & $\begin{array}{l}\text { Mastoiditis, } \\
\text { sinusitis, S. } \\
\text { pneumoniae }\end{array}$ \\
\hline \multirow[t]{4}{*}{$\begin{array}{l}\text { Eggerstedt [2019] } \\
{[58]}\end{array}$} & Spontaneous & Left temporal & $\begin{array}{l}\text { Confusion, aphasia, otalgia, aural } \\
\text { fullness }\end{array}$ & Mastoid & \\
\hline & Spontaneous & $\begin{array}{l}\text { Intraventricular, right } \\
\text { middle cranial fossa }\end{array}$ & Altered mental status & Tegmen tympani & \\
\hline & Spontaneous & Intraventricular & Headache, nausea, photophobia & $\begin{array}{l}\text { Tegmen tympani, } \\
\text { mastoid }\end{array}$ & \\
\hline & Spontaneous & Left frontoparietal & Otalgia, hearing loss, tinnitus & Tegmen tympani & \\
\hline Harth [2019] [59] & Spontaneous & Intraventricular & $\begin{array}{l}\text { Balance problems, tinnitus, aural } \\
\text { fullness, rhinorrhoea, weakness } \\
\text { of right lower limb }\end{array}$ & Petrous bone & CSF leak \\
\hline $\begin{array}{l}\text { Pollaers [2019] } \\
{[60]}\end{array}$ & $\begin{array}{l}\text { Barotrauma } \\
\text { (air travel) }\end{array}$ & Extradural & $\begin{array}{l}\text { Headache, tinnitus, hearing loss, } \\
\text { nasal obstruction, rhinorrhoea, } \\
\text { postnasal drip }\end{array}$ & Mastoid & CSF leak \\
\hline $\begin{array}{l}\text { Voldřich [2019] } \\
\text { [10] }\end{array}$ & Politzer manoeuvre & Middle cranial fossa & $\begin{array}{l}\text { Vertigo, tinnitus, instability, } \\
\text { aphasia }\end{array}$ & Tegmen tympani & $\begin{array}{l}\text { Bilateral } \\
\text { spontaneous } \\
\text { pneumocephalus }\end{array}$ \\
\hline $\begin{array}{l}\text { Stewart [2018] } \\
{[15]}\end{array}$ & $\begin{array}{l}\text { Barotrauma } \\
\text { (air travel) }\end{array}$ & $\begin{array}{l}\text { Right middle cranial } \\
\text { fossa }\end{array}$ & $\begin{array}{l}\text { Vertigo, nausea, vomiting, } \\
\text { headache, otalgia }\end{array}$ & $\begin{array}{l}\text { Tegmen tympani, } \\
\text { petrous }\end{array}$ & \\
\hline Wu [2018] [61] & $\begin{array}{l}\text { Valsalva } \\
\text { manoeuvre } \\
\text { (sneezing) }\end{array}$ & Left occipital lobe & $\begin{array}{l}\text { Headache, dizziness, epistaxis, } \\
\text { tinnitus, otorrhoea }\end{array}$ & Mastoid & $\begin{array}{l}\text { CSF leak; subdural } \\
\text { haemorrhage, } \\
\text { maxillary } \\
\text { hemosinus }\end{array}$ \\
\hline Young [2018] [62] & Spontaneous & $\begin{array}{l}\text { Intraventricular, right } \\
\text { temporal lobe }\end{array}$ & $\begin{array}{l}\text { Headache, rhinorrhoea, acute } \\
\text { lethargy }\end{array}$ & Temporal bone & CSF leak \\
\hline Arai [2017] [63] & Spontaneous & Intraventricular & $\begin{array}{l}\text { Incontinence, dysarthria, } \\
\text { imbalance, ear fullness }\end{array}$ & Petrous bone & $\begin{array}{l}\text { Bilateral } \\
\text { pneumocephalus }\end{array}$ \\
\hline $\begin{array}{l}\text { Guleria [2017] } \\
{[64]}\end{array}$ & Spontaneous & Subarachnoid & $\begin{array}{l}\text { Headache, vomiting, neck } \\
\text { rigidity, otorrhoea }\end{array}$ & Chronic otitis media & \\
\hline $\begin{array}{l}\text { Wannemuehler } \\
{[2016][65]}\end{array}$ & Spontaneous & Frontal lobes & Anosmia, headaches, confusion & Tegmen tympani & 'Mount Fuji sign' \\
\hline Odani [2015] [66] & Spontaneous & Intracranial & Dizziness, fever & Gas forming bacteria & $\begin{array}{l}\text { Streptococcus } \\
\text { pneumoniae }\end{array}$ \\
\hline Ratre [2015] [67] & Spontaneous & $\begin{array}{l}\text { Left cerebellopontine } \\
\text { angle }\end{array}$ & $\begin{array}{l}\text { Fever, headache, vomiting, } \\
\text { decreased hearing, neck rigidity }\end{array}$ & Chronic otitis media & \\
\hline Ginat [2014] [68] & $\begin{array}{l}\text { Barotrauma } \\
\text { (air travel) }\end{array}$ & Intracranial & Dizziness, hearing loss & $\begin{array}{l}\text { Dehiscent right } \\
\text { superior semi- } \\
\text { circular canal }\end{array}$ & \\
\hline $\begin{array}{l}\text { Pishbin [2014] } \\
{[69]}\end{array}$ & $\begin{array}{l}\text { Valsalva } \\
\text { manoeuvre } \\
\text { (coughing) }\end{array}$ & Intracranial & Headache, nausea & $\begin{array}{l}\text { Pneumosinus } \\
\text { dilatans }\end{array}$ & \\
\hline $\begin{array}{l}\text { Remenschneider } \\
\text { [2014] [70] }\end{array}$ & $\begin{array}{l}\text { Barotrauma (air } \\
\text { travel) }\end{array}$ & Extradural & Vertigo, hearing loss, & $\begin{array}{l}\text { Superior canal } \\
\text { dehiscence }\end{array}$ & \\
\hline $\begin{array}{l}\text { Rabello [2013] } \\
\text { [71] }\end{array}$ & $\begin{array}{l}\text { Valsalva } \\
\text { manoeuvre }\end{array}$ & Extradural parietal & $\begin{array}{l}\text { Aural fullness, nasal obstruction, } \\
\text { nasal pruritus, headaches }\end{array}$ & Mastoid & \\
\hline $\begin{array}{l}\text { Tamura [2012] } \\
\text { [72] }\end{array}$ & Spontaneous & Intraventricular & Otalgia, otorrhoea, headache & $\begin{array}{l}\text { Superior external } \\
\text { auditory canal, } \\
\text { mastoid }\end{array}$ & Brain abscess \\
\hline Javan [2011] [73] & $\begin{array}{l}\text { Barotrauma (air } \\
\text { travel) }\end{array}$ & Cisternal & $\begin{array}{l}\text { Confusion, fever, nausea, } \\
\text { vomiting, weakness, headache }\end{array}$ & Bony defect & $\begin{array}{l}\text { Air-fluid level in } \\
\text { right sphenoid } \\
\text { sinus }\end{array}$ \\
\hline
\end{tabular}


Table 1 cont. Non-traumatic pneumocephalus: studies included in review

\begin{tabular}{|c|c|c|c|c|c|}
\hline \multicolumn{6}{|c|}{ Spontaneous otogenic pneumocephalus } \\
\hline Authors [year] & Aetiology & Location & Symptoms & $\begin{array}{l}\text { Location } \\
\text { of air entry }\end{array}$ & Other \\
\hline $\begin{array}{l}\text { Mohammed } \\
\text { [2011] [74] }\end{array}$ & $\begin{array}{l}\text { Valsalva } \\
\text { manoeuvre } \\
\text { (sneezing, nose } \\
\text { blowing) }\end{array}$ & Extradural & Headache & Temporal bone & $\begin{array}{l}\text { Subcutaneous } \\
\text { emphysema }\end{array}$ \\
\hline Zhao [2011] [75] & Spontaneous & Epidural & $\begin{array}{l}\text { Mass in left occipital region, } \\
\text { bilateral hearing deficits }\end{array}$ & Tegmen tympani & $\begin{array}{l}\text { Tension } \\
\text { pneumocephalus, } \\
\text { occipital } \\
\text { subcutaneous } \\
\text { emphysema }\end{array}$ \\
\hline Lee $[2010][76]$ & Spontaneous & Multifocal & Headache, nausea & $\begin{array}{l}\text { Pneumosinus } \\
\text { dilatans }\end{array}$ & \\
\hline $\begin{array}{l}\text { Murugesan } \\
{[2010][77]}\end{array}$ & $\begin{array}{l}\text { Barotrauma (scuba } \\
\text { diving) }\end{array}$ & Extradural & Headache, nausea, vomiting & Ethmoid & \\
\hline Abbati [2009] [78] & Spontaneous & Left temporal lobe & Otalgia, aphasia, headache & $\begin{array}{l}\text { Tegmen tympani, } \\
\text { temporal bone }\end{array}$ & \\
\hline $\begin{array}{l}\text { Pennings [2009] } \\
\text { [79] }\end{array}$ & Spontaneous & Extradural & ND & Mastoid & \\
\hline $\begin{array}{l}\text { Roberts [2009] } \\
{[18]}\end{array}$ & Spontaneous & Intraventricular & $\begin{array}{l}\text { Confusion, rhinorrhoea, urinary } \\
\text { incontinence }\end{array}$ & $\begin{array}{l}\text { Fistula - right } \\
\text { sphenoethmoidal } \\
\text { recess, subsequently } \\
\text { localised to right } \\
\text { sphenoid sinus }\end{array}$ & CSF leak \\
\hline Singh [2009] [9] & $\begin{array}{l}\text { Valsalva } \\
\text { manoeuvre }\end{array}$ & $\begin{array}{l}\text { Left middle cranial } \\
\text { fossa }\end{array}$ & Confusion & $\begin{array}{l}\text { Tegmen tympani, } \\
\text { temporal bone }\end{array}$ & \\
\hline Hyam [2008] [80] & Spontaneous & $\begin{array}{l}\text { Right middle cranial } \\
\text { fossa, intraventricular }\end{array}$ & Confusion, aphasia & $\begin{array}{l}\text { Mastoid, tegmen } \\
\text { tympani }\end{array}$ & \\
\hline $\begin{array}{l}\text { Mathai [2008] } \\
\text { [81] }\end{array}$ & Spontaneous & Intracranial & $\begin{array}{l}\text { Fever, headache, vomiting, } \\
\text { hearing loss }\end{array}$ & $\begin{array}{l}\text { Chronic otitis media; } \\
\text { 'gas forming' bacteria }\end{array}$ & \\
\hline Tucker [2008] [19] & $\begin{array}{l}\text { Valsalva } \\
\text { manoeuvre } \\
\text { (nose blowing) }\end{array}$ & Epidural & Headache, nausea & Mastoid & \\
\hline Villa [2008] [17] & Spontaneous & $\begin{array}{l}\text { Parenchymal (left } \\
\text { temporal lobe) }\end{array}$ & $\begin{array}{l}\text { Abnormal acoustic sensation, } \\
\text { aphasia, visual field disturbances }\end{array}$ & Mastoid & \\
\hline $\begin{array}{l}\text { Bhattacharyya } \\
\text { [2007] [82] }\end{array}$ & Spontaneous & Intracranial & Headache & $\begin{array}{l}\text { Pneumosinus } \\
\text { dilatans of frontal } \\
\text { sinus }\end{array}$ & \\
\hline Ciorba [2007] [83] & Spontaneous & Intracranial & $\begin{array}{l}\text { Otalgia, headache, fever, } \\
\text { otorrhoea, lethargy, nuchal } \\
\text { rigidity, aphasia }\end{array}$ & Acute otitis media & Meningitis \\
\hline $\begin{array}{l}\text { Wilkinson [2007] } \\
\text { [13] }\end{array}$ & $\begin{array}{l}\text { Barotrauma (air } \\
\text { travel) }\end{array}$ & Intraventricular & $\begin{array}{l}\text { Tinnitus, hearing loss, aural } \\
\text { fullness }\end{array}$ & Tegmen tympani & $\begin{array}{l}\text { Temporal lobe } \\
\text { fluid collection }\end{array}$ \\
\hline Hage [2005] [11] & Change in altitude & $\begin{array}{l}\text { Intraparenchymal, } \\
\text { subdural, } \\
\text { intraventricular }\end{array}$ & $\begin{array}{l}\text { Abnormal acoustic sensations, } \\
\text { deterioration, headache }\end{array}$ & Temporal bone & \\
\hline $\begin{array}{l}\text { Krayenbühl } \\
\text { [2005] [16] }\end{array}$ & Spontaneous & Left temporal lobe & $\begin{array}{l}\text { Abnormal acoustic sensations, } \\
\text { aphasia, visual field disturbances }\end{array}$ & Mastoid & $\begin{array}{l}\text { With intracerebral } \\
\text { haemorrhage }\end{array}$ \\
\hline $\begin{array}{l}\text { Jensen }[2004] \\
{[84]}\end{array}$ & $\begin{array}{l}\text { Barotrauma } \\
\text { (air travel) }\end{array}$ & Intraventricular & Headache, tinnitus & Mastoid & \\
\hline \multirow[t]{2}{*}{$\begin{array}{l}\text { Richards [2004] } \\
\text { [8] }\end{array}$} & $\begin{array}{l}\text { Valsalva } \\
\text { manoeuvre } \\
\text { (nose blowing) }\end{array}$ & Extradural & Swelling in ear area, otalgia & Mastoid & $\begin{array}{l}\text { With collection of } \\
\text { subcutaneous air }\end{array}$ \\
\hline & $\begin{array}{l}\text { Valsalva mano- } \\
\text { euvre (coughing, } \\
\text { sneezing, nose } \\
\text { blowing) }\end{array}$ & Extradural & $\begin{array}{l}\text { Pain and paraesthesia over left } \\
\text { cheek and lower jaw }\end{array}$ & Mastoid & \\
\hline
\end{tabular}


Table 1 cont. Non-traumatic pneumocephalus: studies included in review

\begin{tabular}{|c|c|c|c|c|c|}
\hline \multicolumn{6}{|c|}{ Spontaneous otogenic pneumocephalus } \\
\hline Authors [year] & Aetiology & Location & Symptoms & $\begin{array}{l}\text { Location } \\
\text { of air entry }\end{array}$ & Other \\
\hline $\begin{array}{l}\text { Schrijver [2003] } \\
\text { [85] }\end{array}$ & $\begin{array}{l}\text { Valsalva } \\
\text { manoeuvre }\end{array}$ & Epidural & Asymptomatic & Mastoid & \\
\hline $\begin{array}{l}\text { Añorbe [2000] } \\
{[86]}\end{array}$ & $\begin{array}{l}\text { Valsalva } \\
\text { manoeuvre }\end{array}$ & Right parietooccipital & Retroauricular swelling & Mastoid, petrous & $\begin{array}{l}\text { With } \\
\text { pneumatocele }\end{array}$ \\
\hline Babl [1999] [87] & $\begin{array}{l}\text { Valsalva } \\
\text { manoeuvre }\end{array}$ & Subarachnoid & Headache & Sphenoid sinus & \\
\hline Vallejo [1999] [14] & $\begin{array}{l}\text { Valsalva } \\
\text { manoeuvre }\end{array}$ & $\begin{array}{l}\text { Right temporo- } \\
\text { occipital }\end{array}$ & Headache & Mastoid & \\
\hline Dowd [1998] [12] & Spontaneous & $\begin{array}{l}\text { Intraventricular, left } \\
\text { temporal cyst }\end{array}$ & $\begin{array}{l}\text { Headaches, confusion, } \\
\text { forgetfulness, aphasia, } \\
\text { hemiparesis }\end{array}$ & Tegmen tympani & $\begin{array}{l}\text { Abnormally low } \\
\text { ICP }\end{array}$ \\
\hline Maier [1996] [7] & $\begin{array}{l}\text { Valsalva } \\
\text { manoeuvre }\end{array}$ & $\begin{array}{l}\text { Right parieto- } \\
\text { temporo-occipital } \\
\text { region, epidural }\end{array}$ & Headache, visual scotoma & Mastoid & \\
\hline \multicolumn{6}{|c|}{ Pneumocephalus associated with bone defects } \\
\hline $\begin{array}{l}\text { Boninsegna } \\
{[2019][88]}\end{array}$ & $\begin{array}{l}\text { Valsalva } \\
\text { manoeuvre (nose } \\
\text { blowing) }\end{array}$ & $\begin{array}{l}\text { Subdural, } \\
\text { intraventricular }\end{array}$ & Headaches & Cribriform plate & \\
\hline Baba [2016] [23] & $\begin{array}{l}\text { Valsalva } \\
\text { manoeuvre } \\
\text { (sneezing) }\end{array}$ & $\begin{array}{l}\text { Extraaxial air (right } \\
\text { cerebral hemisphere) }\end{array}$ & Headache, rhinorrhoea & Sphenoid sinus & $\begin{array}{l}\text { CSF leak, CSF } \\
\text { collection }\end{array}$ \\
\hline $\begin{array}{l}\text { Mirone [2015] } \\
{[22]}\end{array}$ & $\begin{array}{l}\text { Valsalva } \\
\text { manoeuvre (nose } \\
\text { blowing) }\end{array}$ & $\begin{array}{l}\text { Intraparenchymal } \\
\text { (left frontal lobe) }\end{array}$ & $\begin{array}{l}\text { Loss of consciousness, } \\
\text { hemiparesis, anosmia }\end{array}$ & Ethmoid sinus & \\
\hline $\begin{array}{l}\text { Gaberel [2012] } \\
{[89]}\end{array}$ & Spontaneous & $\begin{array}{l}\text { Intraventricular, } \\
\text { intraparenchymal }\end{array}$ & Confusion & Sphenoid sinus & Arachnoid cyst \\
\hline Nash [2012] [90] & Spontaneous & $\begin{array}{l}\text { Intraparenchymal } \\
\text { (right frontal lobe) }\end{array}$ & Alien limb syndrome & Cribriform plate & \\
\hline $\begin{array}{l}\text { Lefranc [2009] } \\
\text { [20] }\end{array}$ & Spontaneous & $\begin{array}{l}\text { Subarachnoid, } \\
\text { intraventricular }\end{array}$ & $\begin{array}{l}\text { Rhinorrhoea, headaches, } \\
\text { epistaxis, anosmia }\end{array}$ & Cribriform plate & $\begin{array}{l}\text { CSF leak; tension } \\
\text { pneumocephalus }\end{array}$ \\
\hline $\begin{array}{l}\text { Tedeschi [2007] } \\
{[21]}\end{array}$ & Spontaneous & $\begin{array}{l}\text { Intraventricular, } \\
\text { intraparenchymal }\end{array}$ & $\begin{array}{l}\text { Headache, vomiting, fever, } \\
\text { rhinorrhoea, nuchal rigidity, } \\
\text { nystagmus }\end{array}$ & Sphenoid sinus & CSF leak \\
\hline Kuo [2005] [25] & Spontaneous & Subarachnoid & $\begin{array}{l}\text { Headache, rhinorrhoea, neck } \\
\text { rigidity }\end{array}$ & Sphenoid sinus & $\begin{array}{l}\text { Tension } \\
\text { pneumocephalus, } \\
\text { CSF leakage }\end{array}$ \\
\hline Hogg [1998] [91] & Spontaneous & Intracranial & $\begin{array}{l}\text { Rhinorrhoea, left hemiparesis, } \\
\text { headache }\end{array}$ & Sphenoid sinus & \\
\hline Park [1998] [24] & Spontaneous & Epidural & Headaches & Bone defect & $\begin{array}{l}\text { Hyperpneumatisa- } \\
\text { tion of cranium }\end{array}$ \\
\hline \multicolumn{6}{|c|}{ Pneumocephalus associated with malformations } \\
\hline Kutty [2018] [28] & Spontaneous & Intraventricular & $\begin{array}{l}\text { Apnoea, muscular hypotonia in } \\
\text { lower limbs and sphincter }\end{array}$ & $\begin{array}{l}\text { Open } \\
\text { myelomeningocele }\end{array}$ & CSF leak \\
\hline Erol [2004] [29] & Spontaneous & Infratentorial area & Apnoea attacks & $\begin{array}{l}\text { Open lumbosacral } \\
\text { myelomeningocele }\end{array}$ & CSF leak \\
\hline $\begin{array}{l}\text { Oedemis [2004] } \\
{[27]}\end{array}$ & Spontaneous & Intraventricular & Lower limb paralysis & $\begin{array}{l}\text { Open lumbosacral } \\
\text { myelomeningocele }\end{array}$ & $\begin{array}{l}\text { CSF leak; with } \\
\text { infection }\end{array}$ \\
\hline $\begin{array}{l}\text { Garonzik [2001] } \\
\text { [30] }\end{array}$ & Spontaneous & Posterior fossa & $\begin{array}{l}\text { Open lumbosacral } \\
\text { myelomeningocele }\end{array}$ & CSF leak & \\
\hline $\begin{array}{l}\text { Trawöger [1994] } \\
\text { [26] }\end{array}$ & Spontaneous & Intraventricular & Respiratory distress & $\begin{array}{l}\text { Open lumbosacral } \\
\text { myelomeningocele }\end{array}$ & $\begin{array}{l}\text { CSF leak; with } \\
\text { infection }\end{array}$ \\
\hline
\end{tabular}


Table 1 cont. Non-traumatic pneumocephalus: studies included in review

\begin{tabular}{|c|c|c|c|c|c|}
\hline \multicolumn{6}{|c|}{ Pneumocephalus associated with infections } \\
\hline Authors [year] & Aetiology & Location & Symptoms & $\begin{array}{l}\text { Location } \\
\text { of air entry }\end{array}$ & Other \\
\hline Mirzai [2019] [92] & Spontaneous & Intracranial & $\begin{array}{l}\text { Abdominal pain, weakness, } \\
\text { confusion, constipation }\end{array}$ & $\begin{array}{l}\text { Gas-forming } \\
\text { Clostridium } \\
\text { septicum }\end{array}$ & DM type 1 \\
\hline $\begin{array}{l}\text { Saleem [2019] } \\
\text { [93] }\end{array}$ & Spontaneous & Intracranial & $\begin{array}{l}\text { Confusion, disorientation, fever, } \\
\text { headache, vomiting }\end{array}$ & Bacterial infection & $\begin{array}{l}\text { Sinusitis, } \\
\text { pansinusitis, } \\
\text { subdural empyema }\end{array}$ \\
\hline \multirow[t]{2}{*}{ Sun [2019] [94] } & Spontaneous & Intracranial & $\begin{array}{l}\text { Headache, nasal congestion, } \\
\text { vertigo }\end{array}$ & $\begin{array}{l}\text { Gas-forming } \\
\text { Haemophilus } \\
\text { influenzae }\end{array}$ & Mucopyocele \\
\hline & Spontaneous & Intracranial & $\begin{array}{l}\text { Fever, frontal headaches, cough, } \\
\text { rhinorrhoea }\end{array}$ & $\begin{array}{l}\text { Gas-forming } \\
\text { Staphylococcus } \\
\text { aureus and } \\
\text { Escherichia coli }\end{array}$ & Meningitis \\
\hline Ansari [2018] [95] & Spontaneous & Intracranial & Headache, lethargy, deterioration & $\begin{array}{l}\text { Gas-forming } \\
\text { Streptococcus } \\
\text { salivarius }\end{array}$ & $\begin{array}{l}\text { CPAP use } \\
\text { complication, sinus } \\
\text { wall osteomyelitis }\end{array}$ \\
\hline $\begin{array}{l}\text { Kumari [2017] } \\
{[96]}\end{array}$ & Spontaneous & $\begin{array}{l}\text { Subdural and } \\
\text { subgaleal }\end{array}$ & $\begin{array}{l}\text { Refusal to feed, progressive } \\
\text { increase in head size, } \\
\text { hypothermic }\end{array}$ & $\begin{array}{l}\text { Possible bacterial } \\
\text { infection }\end{array}$ & $\begin{array}{l}\text { Neonatal } \\
\text { meningitis, 'air } \\
\text { bubble sign' }\end{array}$ \\
\hline $\begin{array}{l}\text { Srikumar [2017] } \\
\text { [97] }\end{array}$ & Spontaneous & Intracranial & $\begin{array}{l}\text { Left arm clumsiness, altered } \\
\text { mental status, fever }\end{array}$ & Aspergillus terreus & Angiosarcoma \\
\hline $\operatorname{Lin}[2014][98]$ & Spontaneous & Intracranial & Fever, confusion, anisocoria & $\begin{array}{l}\text { Bacterial meningitis } \\
\text { with gas-forming } \\
\text { organisms }\end{array}$ & Air embolism \\
\hline $\begin{array}{l}\text { Subasree [2014] } \\
\text { [99] }\end{array}$ & Spontaneous & Intracranial & Headache, fever, neck rigidity & $\begin{array}{l}\text { Gas-forming } \\
\text { Bacteroides fragilis }\end{array}$ & \\
\hline $\operatorname{Kim}[2013][31]$ & Spontaneous & Intraventricular & $\begin{array}{l}\text { Night sweats, confusion, } \\
\text { headache }\end{array}$ & $\begin{array}{l}\text { Streptococcus } \\
\text { pneumoniae }\end{array}$ & Meningitis \\
\hline $\begin{array}{l}\text { Kosac [2013] } \\
{[100]}\end{array}$ & Spontaneous & Intracranial & $\begin{array}{l}\text { Headache, vomiting, persistent } \\
\text { aqueous rhinorrhoea }\end{array}$ & $\begin{array}{l}\text { Opacification of left } \\
\text { sphenoid sinus and } \\
\text { bone defect of left } \\
\text { pterygoid process }\end{array}$ & Chronic sinusitis \\
\hline Rota [2013] [101] & Spontaneous & Intracranial & Comatose state, fever & $\begin{array}{l}\text { Gas-forming } \\
\text { Streptococcus } \\
\text { pneumoniae }\end{array}$ & $\begin{array}{l}\text { Pneumococcal } \\
\text { meningitis, right } \\
\text { mastoiditis and left } \\
\text { ethmoid sinusitis }\end{array}$ \\
\hline $\begin{array}{l}\text { Shenoi [2013] } \\
\text { [37] }\end{array}$ & Spontaneous & $\begin{array}{l}\text { Subarachnoid, } \\
\text { intraparenchymal, } \\
\text { intravascular }\end{array}$ & $\begin{array}{l}\text { Hypothermia, lethargy, feeding } \\
\text { difficulty }\end{array}$ & Citrobacter koseri & $\begin{array}{l}\text { Late-onset } \\
\text { neonatal } \\
\text { Citrobacter } \\
\text { meningitis }\end{array}$ \\
\hline Baig [2012] [32] & Spontaneous & Subarachnoid space & $\begin{array}{l}\text { Headache, rhinorrhoea, } \\
\text { vomiting, fever, meningeal signs, } \\
\text { bilateral papilledema }\end{array}$ & $\begin{array}{l}\text { Bony erosion around } \\
\text { olfactory cleft }\end{array}$ & Nasal tuberculosis \\
\hline Kaur [2012] [102] & Spontaneous & Subdural & $\begin{array}{l}\text { Fever, headache, convulsions, } \\
\text { neck rigidity, positive Kernig sign }\end{array}$ & Meningitis & \\
\hline $\begin{array}{l}\text { Bhogal }[2011] \\
{[103]}\end{array}$ & Spontaneous & Intraparenchymal & $\begin{array}{l}\text { Diarrhoea, vomiting, altered level } \\
\text { of consciousness, generalised } \\
\text { weakness }\end{array}$ & $\begin{array}{l}\text { Gas-forming } \\
\text { Clostridium } \\
\text { septicum }\end{array}$ & Cerebritis \\
\hline $\begin{array}{l}\text { Kumari }[2011] \\
{[104]}\end{array}$ & Spontaneous & $\begin{array}{l}\text { Intraparenchymal, } \\
\text { intraventricular }\end{array}$ & $\begin{array}{l}\text { Abnormal behaviour, fever, } \\
\text { headache, back and neck pain } \\
\text { vomiting, coughing }\end{array}$ & $\begin{array}{l}\text { Gas-forming } \\
\text { Staphylococcus } \\
\text { aureus }\end{array}$ & $\begin{array}{l}\text { Staphylococcal } \\
\text { pneumonia and } \\
\text { meningitis }\end{array}$ \\
\hline Martin [2011] [35] & Spontaneous & Intraparenchymal & Anuria, bloody diarrhoea & $\begin{array}{l}\text { Clostridium } \\
\text { septicum gas - } \\
\text { producing bacteria }\end{array}$ & $\begin{array}{l}\text { Haemolytic uremic } \\
\text { syndrome }\end{array}$ \\
\hline
\end{tabular}


Table 1 cont. Non-traumatic pneumocephalus: studies included in review

\begin{tabular}{|c|c|c|c|c|c|}
\hline \multicolumn{6}{|c|}{ Pneumocephalus associated with infections } \\
\hline Authors [year] & Aetiology & Location & Symptoms & $\begin{array}{l}\text { Location } \\
\text { of air entry }\end{array}$ & Other \\
\hline Oh [2011] [105] & Spontaneous & Intracranial & Fever, fatigue & $\begin{array}{l}\text { Bony erosion, } \\
\text { Tuberculosis } \\
\text { meningoencephalitis }\end{array}$ & $\begin{array}{l}\text { Lupus } \\
\text { erythematosus }\end{array}$ \\
\hline Redhu [2011] [33] & Spontaneous & Intracranial & $\begin{array}{l}\text { Headache, dizziness, fever, } \\
\text { unconscious with tonic extensor } \\
\text { response to pain }\end{array}$ & Escherichia coli & $\begin{array}{l}\text { Subdural } \\
\text { empyema }\end{array}$ \\
\hline $\begin{array}{l}\text { Damergis [2010] } \\
{[106]}\end{array}$ & Spontaneous & Intracranial & $\begin{array}{l}\text { Headache, photophobia, nausea, } \\
\text { jaw discomfort, subjective fever }\end{array}$ & $\begin{array}{l}\text { Streptococcus } \\
\text { pneumoniae }\end{array}$ & $\begin{array}{l}\text { Otogenic } \\
\text { meningitis }\end{array}$ \\
\hline $\begin{array}{l}\text { Lasboo }[2010] \\
{[107]}\end{array}$ & Spontaneous & Subarachnoid & $\begin{array}{l}\text { Altered mental status, urinary } \\
\text { incontinence, diarrhoea, lower } \\
\text { back pain }\end{array}$ & $\begin{array}{l}\text { Gas-forming } \\
\text { Escherichia coli }\end{array}$ & $\begin{array}{l}\text { Septic discitis, } \\
\text { meningitis, solid } \\
\text { organ abscesses, } \\
\text { urosepsis }\end{array}$ \\
\hline $\begin{array}{l}\text { Chung [2009] } \\
{[108]}\end{array}$ & Spontaneous & Subarachnoid & $\begin{array}{l}\text { Right hypertonia, hyperreflexia, } \\
\text { confusion, dysphasia, right facial } \\
\text { droop }\end{array}$ & $\begin{array}{l}\text { Fistula between } \\
\text { subarachnoid space } \\
\text { crania extension of } \\
\text { cervical spine }\end{array}$ & $\begin{array}{l}\text { Nasopharyngeal } \\
\text { carcinoma, } \\
\text { osteoradionecrosis, } \\
\text { osteomyelitis }\end{array}$ \\
\hline $\begin{array}{l}\text { Hama-Amin } \\
\text { [2009] [109] }\end{array}$ & Spontaneous & $\begin{array}{l}\text { Subarachnoid, } \\
\text { intraparenchymal, } \\
\text { intraventricular }\end{array}$ & $\begin{array}{l}\text { Headache, confusion, } \\
\text { somnolentia, meningeal signs }\end{array}$ & $\begin{array}{l}\text { Gram-negative } \\
\text { bacterial meningitis }\end{array}$ & \\
\hline $\operatorname{Lin}[2009][34]$ & Spontaneous & Intracranial & $\begin{array}{l}\text { Headache, vomiting, fever, } \\
\text { nasal congestion, neck stiffness, } \\
\text { meningeal sign }\end{array}$ & $\begin{array}{l}\text { Candida albicans } \\
\text { sphenoid sinusitis }\end{array}$ & $\begin{array}{l}\text { Pus drained from } \\
\text { right sphenoid } \\
\text { cavity }\end{array}$ \\
\hline Kuo [2008] [38] & Spontaneous & Intracranial & Consciousness loss, hypothermia & E. cloacae & $\begin{array}{l}\text { Nosocomial } \\
\text { meningitis }\end{array}$ \\
\hline $\begin{array}{l}\text { Sreejith [2008] } \\
{[110]}\end{array}$ & Spontaneous & Subarachnoid & $\begin{array}{l}\text { Headache, fever, vomiting, ear } \\
\text { discharge, hearing impairment, } \\
\text { meningeal sign }\end{array}$ & $\begin{array}{l}\text { Klebsiella } \\
\text { pneumoniae } \\
\text { meningitis }\end{array}$ & \\
\hline $\begin{array}{l}\text { Alviedo [2006] } \\
{[39]}\end{array}$ & Spontaneous & Intracranial & $\begin{array}{l}\text { Lethargy, apnoea, pallor, } \\
\text { hypothermia }\end{array}$ & Citrobacter koseri & Meningitis \\
\hline $\begin{array}{l}\text { Townend [2005] } \\
\text { [111] }\end{array}$ & Spontaneous & Intraventricular & Confusion, fever & $\begin{array}{l}\text { Streptococcus } \\
\text { pneumoniae }\end{array}$ & Sinusitis \\
\hline $\begin{array}{l}\text { Parmar [2004] } \\
{[112]}\end{array}$ & Spontaneous & Intracranial & $\begin{array}{l}\text { Lower back pain, fever, night } \\
\text { sweats }\end{array}$ & $\begin{array}{l}\text { Gas-forming } \\
\text { Bacteroides fragilis }\end{array}$ & Meningitis \\
\hline $\begin{array}{l}\text { Pooboni }[2004] \\
{[113]}\end{array}$ & Spontaneous & Intracranial & $\begin{array}{l}\text { Poor feeding, state of collapse, } \\
\text { hypothermia, hypotension }\end{array}$ & $\begin{array}{l}\text { Gas-forming } \\
\text { Citrobacter koseri }\end{array}$ & Pneumatosis oculi \\
\hline $\begin{array}{l}\text { Sedaghatian } \\
\text { [2004] [114] }\end{array}$ & Spontaneous & Intracranial & Fever, irritability, convulsions & $\begin{array}{l}\text { Gas-forming } \\
\text { Enterobacter cloacae }\end{array}$ & $\begin{array}{l}\text { Enterobacter } \\
\text { cloacae septicemia } \\
\text { and neonatal } \\
\text { meningitis }\end{array}$ \\
\hline $\begin{array}{l}\text { Kassim [2003] } \\
{[115]}\end{array}$ & Spontaneous & Intracranial & $\begin{array}{l}\text { Poor feeding, lethargy, } \\
\text { hypotensive, generalised } \\
\text { convulsions }\end{array}$ & $\begin{array}{l}\text { Gas-forming Proteus } \\
\text { mirabilis }\end{array}$ & Meningitis \\
\hline $\begin{array}{l}\text { Goyal [1996] } \\
{[116]}\end{array}$ & Spontaneous & Intracranial & $\begin{array}{l}\text { Fever, hypotensive, altered } \\
\text { sensorium }\end{array}$ & $\begin{array}{l}\text { Gas-forming } \\
\text { Clostridium } \\
\text { perfringens }\end{array}$ & Meningitis \\
\hline $\begin{array}{l}\text { Randall [1993] } \\
{[117]}\end{array}$ & Spontaneous & Intracranial & $\begin{array}{l}\text { Bloody diarrhoea, abdominal } \\
\text { pain, seizure, coma }\end{array}$ & $\begin{array}{l}\text { Gas-forming } \\
\text { Clostridium } \\
\text { septicum }\end{array}$ & $\begin{array}{l}\text { Retinal } \\
\text { haemorrhages }\end{array}$ \\
\hline $\begin{array}{l}\text { Finelli [1991] } \\
{[118]}\end{array}$ & Spontaneous & $\begin{array}{l}\text { Intraventricular, } \\
\text { subarachnoid }\end{array}$ & $\begin{array}{l}\text { Focal left-sided seizure, eye } \\
\text { deviation to left, confusion, } \\
\text { hyperreflexia }\end{array}$ & $\begin{array}{l}\text { Gas-forming } \\
\text { Streptococcus } \\
\text { pneumoniae }\end{array}$ & Meningitis \\
\hline $\begin{array}{l}\text { Candan }[1990] \\
{[36]}\end{array}$ & Spontaneous & Intracranial & $\begin{array}{l}\text { Otorrhoea, fever, nausea, } \\
\text { vomiting, headache, epilepsy } \\
\text { attack, left hemiparesis, neck } \\
\text { stiffness }\end{array}$ & $\begin{array}{l}\text { Gas-forming } \\
\text { organisms: Proteus } \\
\text { mirabilis, Veillonella } \\
\text { spp. }\end{array}$ & $\begin{array}{l}\text { Abscess in right } \\
\text { temporal lobe }\end{array}$ \\
\hline
\end{tabular}


Table 1 cont. Non-traumatic pneumocephalus: studies included in review

\begin{tabular}{|c|c|c|c|c|c|}
\hline \multicolumn{6}{|c|}{ Pneumocephalus associated with infections } \\
\hline Authors [year] & Aetiology & Location & Symptoms & $\begin{array}{l}\text { Location } \\
\text { of air entry }\end{array}$ & Other \\
\hline $\begin{array}{l}\text { Holtby [1990] } \\
{[119]}\end{array}$ & Spontaneous & Intracranial & $\begin{array}{l}\text { Earache, headache, nausea, } \\
\text { vomiting, confusion, neck rigidity }\end{array}$ & $\begin{array}{l}\text { Gas-forming } \\
\text { Streptococcus } \\
\text { pneumoniae }\end{array}$ & \\
\hline Klein [1989] [120] & Spontaneous & $\begin{array}{l}\text { Intraparenchymal, } \\
\text { intraventricular, } \\
\text { subarachnoid }\end{array}$ & $\begin{array}{l}\text { Irregular respiration, } \\
\text { hypotension }\end{array}$ & $\begin{array}{l}\text { Gas-forming } \\
\text { Clostridium } \\
\text { perfringens } \\
\text { meningoencephalitis }\end{array}$ & \\
\hline \multicolumn{6}{|c|}{ Pneumocephalus associated with tumour } \\
\hline $\begin{array}{l}\text { Ghimire [2019] } \\
\text { [50] }\end{array}$ & Spontaneous & Intraventricular & $\begin{array}{l}\text { Mutism, right hemiparesis, } \\
\text { deterioration }\end{array}$ & $\begin{array}{l}\text { Defect in sphenoid } \\
\text { sinus }\end{array}$ & $\begin{array}{l}\text { Ecchordodid } \\
\text { physaliphora (EP) }\end{array}$ \\
\hline $\begin{array}{l}\text { Iplikcioglu [2019] } \\
\text { [121] }\end{array}$ & Spontaneous & Intracranial & Headache, drowsiness, vomiting & $\begin{array}{l}\text { Posterior wall of } \\
\text { frontal sinus }\end{array}$ & $\begin{array}{l}\text { Front ethmoid } \\
\text { osteoma }\end{array}$ \\
\hline $\begin{array}{l}\text { Elabd [2018] } \\
{[122]}\end{array}$ & Spontaneous & Intraventricular & Headache, nausea, rhinorrhoea & $\begin{array}{l}\text { 'One-way valve } \\
\text { mechanism' } \\
\text { spontaneous } \\
\text { to cabergoline } \\
\text { treatment }\end{array}$ & \\
\hline $\begin{array}{l}\text { Albert [2017] } \\
\text { [123] }\end{array}$ & Spontaneous & Intracranial & $\begin{array}{l}\text { Cough, haemoptysis, right } \\
\text { chest and back pain, right arm } \\
\text { weakness }\end{array}$ & $\begin{array}{l}\text { Bronchopleural- } \\
\text { dural fistula }\end{array}$ & $\begin{array}{l}\text { Chemoradiation, } \\
\text { superior sulcus } \\
\text { tumour }\end{array}$ \\
\hline $\begin{array}{l}\text { Hackenbroch } \\
{[2017][124]}\end{array}$ & Spontaneous & $\begin{array}{l}\text { Intraparenchymal, } \\
\text { right frontal lobe }\end{array}$ & $\begin{array}{l}\text { Headache, difficulty with } \\
\text { concentration, left arm weakness }\end{array}$ & $\begin{array}{l}\text { Erosion of posterior } \\
\text { wall of frontal sinus }\end{array}$ & $\begin{array}{l}\text { Sinonasal osteoma, } \\
\text { 'one-way valve } \\
\text { mechanism' }\end{array}$ \\
\hline $\begin{array}{l}\text { Umredkar [2017] } \\
\text { [125] }\end{array}$ & Spontaneous & Intracranial & Headache, left side hemiparesis & $\begin{array}{l}\text { Erosion of posterior } \\
\text { wall of frontal sinus }\end{array}$ & $\begin{array}{l}\text { Frontal sinus } \\
\text { osteoma, 'ball } \\
\text { valve mechanism' }\end{array}$ \\
\hline $\begin{array}{l}\text { Jimenez [2014] } \\
\text { [126] }\end{array}$ & Spontaneous & Intraventricular & $\begin{array}{l}\text { Aqueous rhinorrhoea, altered } \\
\text { mental condition }\end{array}$ & Nasocranial fistula & $\begin{array}{l}\text { Nasopharyngeal } \\
\text { carcinoma, } \\
\text { radiotherapy }\end{array}$ \\
\hline $\begin{array}{l}\text { Marchant [2013] } \\
\text { [127] }\end{array}$ & Spontaneous & $\begin{array}{l}\text { Intraventricular, air in } \\
\text { thoracic spinal canal }\end{array}$ & Headache & $\begin{array}{l}\text { Oesophageal- } \\
\text { arachnoid fistula }\end{array}$ & $\begin{array}{l}\text { Non-small cell lung } \\
\text { carcinoma }\end{array}$ \\
\hline $\begin{array}{l}\text { Nanba [2013] } \\
{[128]}\end{array}$ & Spontaneous & $\begin{array}{l}\text { Intraventricular and } \\
\text { subarachnoid spaces }\end{array}$ & $\begin{array}{l}\text { Headache, vomiting, delirious. } \\
\text { CSF rhinorrhoea }\end{array}$ & $\begin{array}{l}\text { Cabergoline } \\
\text { treatment }\end{array}$ & $\begin{array}{l}\text { MEN-1, macro- } \\
\text { prolactinoma, } \\
\text { treatment with } \\
\text { cabergoline }\end{array}$ \\
\hline Patel [2013] [49] & Spontaneous & Intraventricular & Headache & $\begin{array}{l}\text { Oesophageal- } \\
\text { subarachnoid fistula }\end{array}$ & $\begin{array}{l}\text { Oesophageal } \\
\text { cancer }\end{array}$ \\
\hline $\begin{array}{l}\text { lacoangeli [2012] } \\
\text { [48] }\end{array}$ & Spontaneous & $\begin{array}{l}\text { Subdural (bifrontal), } \\
\text { pneumorrhachis }\end{array}$ & Headache, confusion & Sacral bone & $\begin{array}{l}\text { Colon } \\
\text { adenocarcinoma }\end{array}$ \\
\hline $\begin{array}{l}\text { Lehmer [2012] } \\
\text { [41] }\end{array}$ & Spontaneous & Intracranial & Headache, nausea, vomiting & $\begin{array}{l}\text { Destruction of } \\
\text { posterior wall of } \\
\text { frontal sinus }\end{array}$ & $\begin{array}{l}\text { Osteoma, one-way } \\
\text { valve mechanism }\end{array}$ \\
\hline $\begin{array}{l}\text { Machicado [2012] } \\
\text { [43] }\end{array}$ & Spontaneous & $\begin{array}{l}\text { Subarachnoid, } \\
\text { intraventricular }\end{array}$ & Rhinorrhoea, vomiting, headache & $\begin{array}{l}\text { Cabergoline } \\
\text { treatment }\end{array}$ & $\begin{array}{l}\text { Macroprolacti- } \\
\text { noma }\end{array}$ \\
\hline $\begin{array}{l}\text { Guedes [2011] } \\
{[129]}\end{array}$ & Spontaneous & Intraparenchymal & $\begin{array}{l}\text { Headache, right hemiparesis, } \\
\text { seventh cranial nerve palsy }\end{array}$ & Bone erosion & $\begin{array}{l}\text { Fronthoethmoidal } \\
\text { osteoma }\end{array}$ \\
\hline Qu [2010] [45] & Spontaneous & $\begin{array}{l}\text { Subdural (bifrontal), } \\
\text { vertebral canal }\end{array}$ & Headache, altered consciousness & $\begin{array}{l}\text { Subarachnoid- } \\
\text { oesophagus fistula }\end{array}$ & $\begin{array}{l}\text { Oesophageal } \\
\text { cancer;'Mount Fuji } \\
\text { sign' }\end{array}$ \\
\hline $\begin{array}{l}\text { Chung [2009] } \\
{[130]}\end{array}$ & Spontaneous & $\begin{array}{l}\text { Intracranial, } \\
\text { subarachnoid }\end{array}$ & $\begin{array}{l}\text { Confusion, expressive dysphasia, } \\
\text { right facial droop, right-sided } \\
\text { hypertonia and hyperreflexia }\end{array}$ & $\begin{array}{l}\text { Osteoradionecrosis } \\
\text { of cervical spine }\end{array}$ & $\begin{array}{l}\text { Nasopharyngeal } \\
\text { cancer, meningitis }\end{array}$ \\
\hline $\begin{array}{l}\text { Kamide [2008] } \\
{[40]}\end{array}$ & Spontaneous & Intraparenchymal & Headache, left hemiparesis & $\begin{array}{l}\text { Erosion of upper wall } \\
\text { of ethmoid sinus }\end{array}$ & $\begin{array}{l}\text { Osteoma in } \\
\text { ethmoid sinus }\end{array}$ \\
\hline
\end{tabular}


Table 1 cont. Non-traumatic pneumocephalus: studies included in review

\begin{tabular}{|c|c|c|c|c|c|}
\hline \multicolumn{6}{|c|}{ Pneumocephalus associated with tumour } \\
\hline Authors [year] & Aetiology & Location & Symptoms & $\begin{array}{l}\text { Location } \\
\text { of air entry }\end{array}$ & Other \\
\hline $\begin{array}{l}\text { Torres [2008] } \\
\text { [131] }\end{array}$ & Spontaneous & Spinal canal & $\begin{array}{l}\text { Tightness in thigh muscles, pain } \\
\text { in perineal area }\end{array}$ & $\begin{array}{l}\text { Gas communication } \\
\text { between carcinoma } \\
\text { of sigmoid colon into } \\
\text { spinal canal }\end{array}$ & $\begin{array}{l}\text { Carcinoma of } \\
\text { sigmoid colon }\end{array}$ \\
\hline Wang [2006] [44] & Spontaneous & $\begin{array}{l}\text { Intraparenchymal, } \\
\text { intraventricular }\end{array}$ & $\begin{array}{l}\text { Headache, vomiting, } \\
\text { blurred vision, rhinorrhoea, } \\
\text { consciousness disturbances }\end{array}$ & $\begin{array}{l}\text { Skull base defect; } \\
\text { high-dose } \\
\text { radiotherapy }\end{array}$ & $\begin{array}{l}\text { Nasopharyngeal } \\
\text { carcinoma }\end{array}$ \\
\hline $\begin{array}{l}\text { Bramley [2001] } \\
{[132]}\end{array}$ & Spontaneous & Intracranial & Left hemiparesis & $\begin{array}{l}\text { Erosion of right } \\
\text { ethmoid sinus wall } \\
\text { and right orbit }\end{array}$ & Ethmoid osteoma \\
\hline Wu [1999] [133] & Spontaneous & $\begin{array}{l}\text { Intraventricular, } \\
\text { intracranial }\end{array}$ & $\begin{array}{l}\text { Nasal discharge, loss of } \\
\text { consciousness }\end{array}$ & $\begin{array}{l}\text { Bony defect in right } \\
\text { sphenoid body }\end{array}$ & $\begin{array}{l}\text { Ventricular } \\
\text { fluid revealed } \\
\text { Staphylococcus } \\
\text { infection }\end{array}$ \\
\hline $\begin{array}{l}\text { Jakubowski } \\
\text { [1997] [134] }\end{array}$ & Spontaneous & Intracranial & $\begin{array}{l}\text { Forgetfulness, left hemiparesis, } \\
\text { anisocoric pupils }\end{array}$ & Bony destruction & $\begin{array}{l}\text { Diploic epidermoid } \\
\text { cyst }\end{array}$ \\
\hline Kiu [1996] [135] & Spontaneous & $\begin{array}{l}\text { Intraventricular, } \\
\text { subarachnoid, } \\
\text { subdural }\end{array}$ & $\begin{array}{l}\text { Headache, rhinorrhoea, } \\
\text { deterioration, neck stiffness }\end{array}$ & $\begin{array}{l}\text { Destruction of skull } \\
\text { base by tumour }\end{array}$ & $\begin{array}{l}\text { Tension } \\
\text { pneumocephalus; } \\
\text { nasopharyngeal } \\
\text { carcinoma, } \\
\text { meningitis }\end{array}$ \\
\hline $\mathrm{Ng}$ [1995] [136] & Spontaneous & Intracranial & $\begin{array}{l}\text { Headache, fever, multiple nerve } \\
\text { palsy }\end{array}$ & $\begin{array}{l}\text { Noso-cranial fistula, } \\
\text { bone necrosis }\end{array}$ & $\begin{array}{l}\text { Nasopharyngeal } \\
\text { carcinoma, } \\
\text { radiotherapy }\end{array}$ \\
\hline $\begin{array}{l}\text { Kinsley [1993] } \\
{[42]}\end{array}$ & Spontaneous & Intracranial & $\begin{array}{l}\text { Left-side weakness, headache, } \\
\text { confusion, drowsiness, decreased } \\
\text { taste and smell }\end{array}$ & Bone defect in sinus & $\begin{array}{l}\text { Epidermoid } \\
\text { tumour of ethmoid } \\
\text { sinus origin }\end{array}$ \\
\hline Swaid [1983] [46] & Spontaneous & $R$ frontal fossa & Headache, neck pain, confusion & $\begin{array}{l}\text { Bronchial- } \\
\text { subarachnoid fistula }\end{array}$ & Lung carcinoma \\
\hline Lynn [1978] [51] & Spontaneous & Intracranial & Fever, irritability, poor feeding & Neurenteric fistula & $\begin{array}{l}\text { Benign teratoma, } \\
\text { meningitis }\end{array}$ \\
\hline $\begin{array}{l}\text { Banerjee [1975] } \\
{[137]}\end{array}$ & Spontaneous & Intracranial & Headache, vomiting & Dural defect & $\begin{array}{l}\text { Osteoid osteoma } \\
\text { of ethmoid }\end{array}$ \\
\hline $\begin{array}{l}\text { Wilson [1968] } \\
{[138]}\end{array}$ & Spontaneous & Intracranial & $\begin{array}{l}\text { Brown-coloured discharge from } \\
\text { left nostril }\end{array}$ & $\begin{array}{l}\text { Destruction of } \\
\text { frontal sinus wall }\end{array}$ & $\begin{array}{l}\text { Epidermoid } \\
\text { tumour }\end{array}$ \\
\hline $\begin{array}{l}\text { Holmes [1957] } \\
{[47]}\end{array}$ & Spontaneous & Intraventricular & $\begin{array}{l}\text { Nasal obstruction, } \\
\text { exophthalmos, headache }\end{array}$ & Cribriform plate & $\begin{array}{l}\text { Sarcoma of } \\
\text { ethmoid }\end{array}$ \\
\hline \multicolumn{6}{|c|}{ Pneumocephalus associated with intravenous air injection } \\
\hline $\begin{array}{l}\text { Laurent }[2014] \\
{[53]}\end{array}$ & Spontaneous & $\begin{array}{l}\text { Right cavernous } \\
\text { sinus, right inferior } \\
\text { ophthalmic vein, left } \\
\text { cavernous sinus, right } \\
\text { sphenoid sinus }\end{array}$ & Asymptomatic & $\begin{array}{l}\text { Peripheral } \\
\text { intravenous catheter }\end{array}$ & $\begin{array}{l}\text { Ripped out } \\
\text { intravenous } \\
\text { catheter }\end{array}$ \\
\hline \multirow[t]{3}{*}{ Tran [2010] [52] } & Spontaneous & $\begin{array}{l}\text { Right cavernous } \\
\text { sinus, right superficial } \\
\text { temporal veins, left } \\
\text { intraorbital veins }\end{array}$ & Asymptomatic & $\begin{array}{l}\text { Peripheral } \\
\text { intravenous catheter }\end{array}$ & \\
\hline & Spontaneous & $\begin{array}{l}\text { Right cavernous } \\
\text { sinus, right superficial } \\
\text { temporal veins, right } \\
\text { superior orbital veins }\end{array}$ & Asymptomatic & $\begin{array}{l}\text { Peripheral } \\
\text { intravenous catheter }\end{array}$ & \\
\hline & Spontaneous & $\begin{array}{l}\text { Bilateral cavernous } \\
\text { sinus, behind dorsum } \\
\text { sella, right superficial } \\
\text { temporal veins }\end{array}$ & Asymptomatic & $\begin{array}{l}\text { Peripheral } \\
\text { intravenous catheter }\end{array}$ & \\
\hline
\end{tabular}


Table 1 cont. Non-traumatic pneumocephalus: studies included in review

\begin{tabular}{|c|c|c|c|c|c|}
\hline \multicolumn{6}{|c|}{ Pneumocephalus associated with intravenous air injection } \\
\hline Authors [year] & Aetiology & Location & Symptoms & $\begin{array}{l}\text { Location } \\
\text { of air entry }\end{array}$ & Other \\
\hline Syed [2008] [54] & Spontaneous & Intracranial & $\begin{array}{l}\text { Visual disturbances, headache, } \\
\text { dizziness }\end{array}$ & $\begin{array}{l}\text { Intravenous } \\
\text { catheterisation }\end{array}$ & Gas emboli \\
\hline \multicolumn{6}{|c|}{ Pneumocephalus associated with other aetiology } \\
\hline Isikay [2018] [55] & Spontaneous & Subarachnoid & Generalised seizures & $\begin{array}{l}\text { Interruption in } \\
\text { cervical dural sheath }\end{array}$ & $\begin{array}{l}\text { Positive end } \\
\text { ventilation }\end{array}$ \\
\hline $\begin{array}{l}\text { Akashi [2015] } \\
{[139]}\end{array}$ & Spontaneous & Intracranial & Headache, nasal hydrorrhoea & Nasal fistula & $\begin{array}{l}\text { Granulomatosis } \\
\text { with polyangiitis }\end{array}$ \\
\hline $\begin{array}{l}\text { Miyaji [2013] } \\
\text { [140] }\end{array}$ & Spontaneous & $\begin{array}{l}\text { Subarachnoid, } \\
\text { intraventricular, } \\
\text { pneumatocele in } \\
\text { spinal canal }\end{array}$ & Headache, fever & $\begin{array}{l}\text { Fistula in sacral } \\
\text { region }\end{array}$ & Pressure ulcer \\
\hline Park [2010] [56] & Spontaneous & Epidural & Headache & $\begin{array}{l}\text { 'One-way valve' } \\
\text { mechanism }\end{array}$ & \\
\hline
\end{tabular}

represent $8.8 \%$ of all cases [3]. Bacterial infections are the most common, and they can cause subdural empyema with meningitis, leading to spontaneous pneumocephalus [31-33]. Two possible pathways were described: (1) an infection with 'gasforming' bacteria and (2) a bony erosion caused by bacteria. Redhu et al. described a case of spontaneous tension pneumocephalus associated with subdural empyema caused typically by Escherichia coli. Pneumocephalus, therefore, resulted from an accumulation of gases in the nerve tissue as a result of bacterial metabolism [33]. Baig et al. presented a case of spontaneous pneumocephalus associated with nasal tuberculosis. As a result of a Mycobacterium tuberculosis infection, bone erosion around the olfactory cleft occurred, with subsequent dura mater damage and CSF leakage [32]. Lin et al. described a case of spontaneous pneumocephalus associated with sphenoid sinusitis caused by Cancida albicans. Extensive fungal osteomyelitis caused bone erosion of the right sphenoid sinus and resulted in intracranial pneumocephalus [34]. Martin et al. presented a case of spontaneous pneumocephalus associated with haemolytic uremic syndrome. As a result of Clostridium septicum-induced sepsis, many pockets of pneumocephalus created a 'Swiss cheese' appearance on brain CT scans [35]. The symptoms are typical for meningitis: headache, fever, vomiting, and night sweats (Tab. 1) [31, 33, 36]. Tension pneumocephalus as a result of bacterial meningitis is usually fatal [37-39].

\section{Pneumocephalus associated with tumours}

A thorough search of the databases revealed a total of 30 articles of pneumocephalus associated with tumours (Tab. 1). Pneumocephalus related to tumours has mostly been associated with sinus osteomas, followed by epidermoids, pituitary tumours, and nasopharyngeal carcinomas [40-44]. Different factors can destroy the tissues surrounding tumour cells, such as fistulae between the posterior wall of the oesophagus and the subarachnoid space of the spinal cord, which contribute to trapping air in the cranial cavity or in subdural spaces [45]. Additionally, pneumocephalus can be caused by radionecrosis as a result of treatment (e.g. subarachnoid bronchial fistulae as a result of irradiation therapy) [44, 46]. Furthermore, tension pneumocephalus can occur spontaneously as a result of treating dopaminergic agents with cabergoline [47, 48]. The main symptoms include headache, rhinorrhoea (presumably CSF), and confusion [45-49]. Interestingly, Torres et al. described a patient with stiffness in their thigh muscles and perineal areas as a result of gas collection in the subdural layer of the sacral nerve root [50]. Lynn et al. presented a case of spontaneous pneumocephalus with neurenteric communications in the form of a neurenteric fistula [51]. Wang et al. presented an interesting case of tension spontaneous pneumocephalus due to intensive high-dose radiotherapy, which caused osteoradionecrosis of the skull base and CSF leakage [44]. Interestingly, Kinsley et al. described a patient with tension pneumatocele originating from an epidermoid tumour. The patient developed symptoms for six months prior to his admission, which shows that the signs of pneumocephalus can develop either severely, within the first 24 hours of occurrence, or slowly progress over weeks or even months [42].

\section{Pneumocephalus associated with an intravenous injection of air}

The database search revealed only three articles on intravenous-related pneumocephalus (Tab. 1). This is considered a rare cause of pneumocephalus, usually related to the placement of an intravenous catheter [52]. Laurent et al. reported a patient with pneumocephalus resulting from an air embolism caused by a ripped-out peripheral intravenous access [53]. Tran et al. suggested that the diagnosis of intravenous-induced pneumocephalus should be considered in every case of incidentally detected air in the cranial cavity venous system on CT scans unless a medical reason is known [52]. In the reported cases, the most common location of pneumocephalus was the sinus cavernous $[52,53]$. Intravenous-related pneumocephalus is associated with a good prognosis [54]. Pneumocephalus associated with an air embolism usually remains asymptomatic $[52,53]$. 


\section{Pneumocephalus associated with other aetiologies}

Four case reports on the occurrence of spontaneous pneumocephalus related to other factors which could not be classified as belonging to the above-mentioned groups, were found in the chosen databases (Tab. 1). Isikay et al. reported an infant with bronchopneumonia who developed pneumocephalus as a result of positive ventilation during treatment for bronchopneumonia [55]. A generalised inflammatory reaction can disrupt the nerve sheaths, which - in combination with positive pressure ventilation - can result in air entering the subarachnoid spaces. Park et al. reported a case of pneumocephalus resulting from pneumatocele in the frontal sinus [56]. Air entered through the frontal sinus ostium but was unable to exit, which increased pressure in the sinus and caused subsequent expansion. The most common symptoms include headache and nausea, but also generalised seizures $[55,56]$.

\section{Discussion}

To the best of our knowledge, ours is the first case report of spontaneous pneumocephalus due to air embolism secondary to lung cancer. In our case, lung cancer caused infiltration of the left atrium and the left ventricle; therefore, air from the left ventricle entered the aorta and then travelled through the brachiocephalicus trunk and carotid arteries into cerebral circulation, causing a massive air embolism.

Spontaneous intravenous pneumocephalus caused by an air embolism is a rare condition - so far, only three cases have been described [51-53]. An air embolism underlying pneumocephalus can affect both the arterial and venous vessels. In contrast to our case, the remaining reports presented pneumocephalus due to venous embolism as a result of intravenous air injection through a peripheral venous catheter [51,52]. Patients with intravenous pneumocephalus can show acute symptoms within the first 24 hours of occurrence, similar to the patient presented here, or can show slowly progressing signs within weeks [47]. The course of the disease in the presented patient was acute, accompanied by disturbances of consciousness and respiratory failure resulting in death. In contrast, Laurent et al. describe a mild, asymptomatic course of pneumocephalus related to intravenous air injection through a peripheral venous catheter [51,52]. However, Syed et al. reported that the patient experienced visual disturbances, headache, and dizziness due to gas embolism during intravenous catheterisation [53].

Our review of the literature confirms that the most common causes of pneumocephalus are otogenic and infectious, followed by tumours. A minority of pneumocephalus cases are caused by intravenous air injection and other cases.

Symptoms and the clinical course of pneumocephalus can vary, depending primarily on aetiology and the location of intracranial gas. The most frequent symptoms, regardless of pneumocephalus aetiology, are headaches and consciousness disturbances $[30,59,99,120]$. In otogenic spontaneous pneumocephalus, otalgia, aural fullness, hearing loss, tinnitus, otorrhoea, and abnormal acoustic sensations are also often described. In pneumocephalus caused by infections, fever and meningeal symptoms are the most common symptoms $[31,92,98,108]$.

\section{References}

1. Paiva WS, de Andrade AF, Figueiredo EG, et al. Effects of hyperbaric oxygenation therapy on symptomatic pneumocephalus. Ther Clin Risk Manag. 2014; 10: 769-773, doi: 10.2147/TCRM.S45220, indexed in Pubmed: 25328392.

2. JELSMA F, MOORE DF. Cranial aerocele. Am J Surg. 1954; 87(3): 437451, doi: 10.1016/s0002-9610(54)90147-6, indexed in Pubmed: 13124659.

3. Markham JW. The clinical features of pneumocephalus based upon a survey of 284 cases with report of 11 additional cases. Acta Neurochir (Wien). 1967; 16(1): 1-78, doi: 10.1007/BF01401900, indexed in Pubmed: 6032371.

4. JELSMA F, MOORE DF. Cranial aerocele. Am J Surg. 1954; 87(3): 437-451, doi: 10.1016/s0002-9610(54)90147-6, indexed in Pubmed: 13124659.

5. HOROWITZ M. Intracranial pneumatocele - an unusual complication following mastoid surgery. J Laryngol Otol. 1964; 78: 128-134, doi: 10.1017/s0022215100061910, indexed in Pubmed: 14126276.

6. DANDY W. Pneumocephalus (intracranial pneumatocele or aerocele). Archives of Surgery. 1926; 12(5): 949, doi: 10.1001/archsurg.1926.01130050003001.

7. Maier W, Fradis M, Scheremet R. Spontaneous otogenic pneumocephalus. Ann Otol Rhinol Laryngol. 1996; 105(4): 300-302, doi: 10.1177/000348949610500411, indexed in Pubmed: 8604893.

8. Richards SD, Saeed SR, Laitt R, et al. Hypercellularity of the mastoid as a cause of spontaneous pneumocephalus. J Laryngol Otol. 2004; 118(6): 474-476, doi: 10.1258/002221504323219653, indexed in Pubmed: 15285871.

9. Singh A, Alvarez J. Spontaneous otogenic intracerebral pneumocephalus. West J Emerg Med. 2010; 11(1): 107, indexed in Pubmed: 20411098

10. Voldřich R, Májovský M, Chovanec M, et al. First Case Report of Bilateral Spontaneous Otogenic Pneumocephalus. World Neurosurg. 2019; 125: 179-182, doi: 10.1016/j.wneu.2019.01.232, indexed in Pubmed: 30771540.

11. Hage P, Daou B, Jabbour P. Spontaneous otogenic pneumocephalus due to altitude changes: $A$ case report and review of literature. Clin Neurol Neurosurg. 2015; 138: 162-164, doi: 10.1016/j.clineuro.2015.08.025, indexed in Pubmed: 26342437.

12. Dowd GC, Molony TB, Voorhies RM. Spontaneous otogenic pneumocephalus. Case report and review of the literature. J Neurosurg. 1998; 89(6): 1036-1039, doi: 10.3171/jns.1998.89.6.1036, indexed in Pubmed: 9833834.

13. Wilkinson EP, Meyer TA, Rubinstein JT. Spontaneous otogenic pneumocephalus managed with the middle fossa approach. Acta Otolaryngol. 2007; 127(8): 892-896, doi: 10.1080/00016480601053081, indexed in Pubmed: 17763004.

14. Vallejo LA, Gil-Carcedo LM, Borrás JM, et al. Spontaneous pneumocephalus of an otogenic origin. Otolaryngol Head Neck Surg. 1999; 121(5): 662-665, doi: 10.1016/S0194-5998(99)70080-X, indexed in Pubmed: 10547494. 
15. Stewart M, Farrell C, Pelosi S. Spontaneous Otogenic Pneumocephalus: Reporting a Unique Site of Dehiscence. ОTO Open. 2019; 3(1): 2473974X18821931, doi: 10.1177/2473974X18821931, indexed in Pubmed: 31236533.

16. Krayenbühl N, Alkadhi H, Jung HH, et al. Spontaneous otogenic intracerebral pneumocephalus: case report and review of the literature. Eur Arch Otorhinolaryngol. 2005; 262(2): 135-138, doi: 10.1007/ s00405-004-0754-8, indexed in Pubmed: 15004711.

17. Villa RA, Capdevila A. Images in clinical medicine. Spontaneous otogenic pneumocephalus. N Engl J Med. 2008; 358(12): e13, doi: 10.1056/NEJMicm063225, indexed in Pubmed: 18354098.

18. Roberts GA, Foy PM, Bolger C. Idiopathic spontaneous cerebrospinal fluid rhinorrhoea and pneumocephalus: case report and literature review. $\mathrm{Br} J$ Neurosurg. 1996; 10(5): 513-517, doi: 10.1080/02688699647195, indexed in Pubmed: 8922715.

19. Tucker A, Miyake H, Tsuji M, et al. Spontaneous epidural pneumocephalus. Neurol Med Chir (Tokyo). 2008; 48(10): 474-478, doi: 10.2176/nmc.48.474, indexed in Pubmed: 18948684.

20. Lefranc M, Peltier J, Demuynkc F, et al. Tension pneumocephalus and rhinorrhea revealing spontaneous cerebrospinal fluid fistula of the anterior cranial base. Neurochirurgie. 2009; 55(3): 340-344, doi: 10.1016/j.neuchi.2008.10.014, indexed in Pubmed: 19359019.

21. Tedeschi E, laccarino C, Covelli EM, et al. MR-Cisternography with T2-Weighted Single-Shot Fast Spin Echo Sequence in the Diagnosis of a Spontaneous CSF Fistula of the Sphenoid Sinus Causing Massive Pneumocephalus. Neuroradiol J. 2009; 21(6): 795-799, doi: 10.1177/197140090802100607, indexed in Pubmed: 24257047.

22. Mirone G, Rotondo M, Scuotto A, et al. Spontaneous intraparenchymal tension pneumocephalus triggered by compulsive forceful nose blowing. Emerg Med J. 2009; 26(11): 837-838, doi: 10.1136/ emj.2008.067124, indexed in Pubmed: 19850819.

23. Baba M, Tarar O, Syed A. A Rare Case of Spontaneous PneumocephaIus Associated with Nontraumatic Cerebrospinal Fluid Leak. Case Rep Neurol Med. 2016; 2016: 1828461, doi: 10.1155/2016/1828461, indexed in Pubmed: 27217961.

24. Park P, Chandler WF, Telian SA, et al. Spontaneous chronic epidural pneumocephalus resulting from hyperpneumatization of the cranium causing mass effect: case report. Neurosurgery. 1998; 42(6): 1384-1386, doi: 10.1097/00006123-199806000-00123, indexed in Pubmed: 9632201.

25. Kuo MY, Lien WC, Wang HP, et al. Nontraumatic tension pneumocephalus--a differential diagnosis of headache at the ED. Am J Emerg Med. 2005; 23(2): 235-236, doi: 10.1016/j.ajem.2004.02.044, indexed in Pubmed: 15765362.

26. Trawöger R, Strasser K, Ellemunter H, et al. Spontaneous pneumocephalus in a newborn infant with myelomeningocele and hydromyelia. Dev Med Child Neurol. 1994; 36(10): 924-927, doi: 10.1111/j.14698749.1994.tb11784.x, indexed in Pubmed: 7926325.

27. Oedemis E, Aslan Y. Spontaneous pneumocephalus associated with open myelomeningocele. Indian Pediatrics. 2004; 41: 289-290.

28. Kutty RK, Sreemathyamma SB, Sivanandapanicker JL, et al. Spontaneous Pneumocephalus Secondary to Leaking Myelomeningocele. J Pediatr Neurosci. 2018; 13(3): 329-330, doi: 10.4103/JPN. JPN_17_18, indexed in Pubmed: 30271466.

29. Erol FS, Kaplan M. Spontaneous pneumocephalus presenting with apnea attacks in a newborn with open myelomeningocele. Pediatr Neurosurg. 2004; 40(6): 312-313, doi: 10.1159/000083746, indexed in Pubmed: 15821364.

30. Garonzik IM, Samdani AF, Carson BS, et al. Pneumocephalus in a newborn with an open myelomeningocele. Pediatr Neurosurg.
2001; 35(6): 334, doi: 10.1159/000050447, indexed in Pubmed: 11786703.

31. Kim HS, Kim SW, Kim SH. Spontaneous pneumocephalus caused by pneumococcal meningitis. J Korean Neurosurg Soc. 2013; 53(4): 249-251, doi: 10.3340/jkns.2013.53.4.249, indexed in Pubmed: 23826483.

32. Baig WW, Nagaraja MV, Varma M. Spontaneous cerebrospinal fluid rhinorrhea with pneumocephalus: an unusual manifestation of nasal tuberculosis. Korean J Intern Med. 2012; 27(3): 350-352, doi: 10.3904/kjim.2012.27.3.350, indexed in Pubmed: 23019402.

33. Redhu R, Shah A, Jadhav M, et al. Spontaneous tension pneumocephalus in a patient with subdural empyema. J Clin Neurosci. 2011; 18(8): 1123-1124, doi: 10.1016/j.jocn.2010.12.029, indexed in Pubmed: 21680188.

34. Lin JJ, Wu CT, Hsia SH, et al. Pneumocephalus: a rare presentation of Candida sphenoid sinusitis. Pediatr Neurol. 2009; 40(5): 398400, doi: 10.1016/j.pediatrneurol.2008.11.016, indexed in Pubmed: 19380081.

35. Martin SE, Allen SD, Faught P, et al. A 2-year-old boy with hemolytic uremic syndrome and pneumocephalus. Brain Pathol. 2012; 22(1): 121-124, doi: 10.1111/j.1750-3639.2011.00555.x, indexed in Pubmed: 22150928.

36. Candan S, Katelioğlu M, Ceylan S, et al. Otogenic brain abscess with pneumocephalus. Infection. 1990; 18(3): 191-192, doi: 10.1007/ BF01642114, indexed in Pubmed: 2365472.

37. Shenoi AN, Shane AL, Fortenberry JD, et al. Spontaneous pneumocephalus in vertically acquired, late-onset neonatal Citrobacter meningitis. J Pediatr. 2013; 163(6): 1791, doi: 10.1016/j.jpeds.2013.08.010, indexed in Pubmed: 24054430.

38. Kuo CC, Wang JY, Chien JY, et al. Nontraumatic pneumocephalus due to nosocomial Enterobacter cloacae infection. Diagn Microbiol Infect Dis. 2010; 66(1): 108-110, doi: 10.1016/j.diagmicrobio.2009.03.024, indexed in Pubmed: 19442474.

39. Alvideo JN, Sood BG, Aranda JV, et al. Diffuse pneumocephalus in neonatal Citrobacter meningitis. Pediatrics. 2006; 118: 1576-1579.

40. Kamide T, Nakada M, Hayashi Y, et al. Intraparenchymal pneumocephalus caused by ethmoid sinus osteoma. J Clin Neurosci. 2009; 16(11): 1487-1489, doi: 10.1016/j.jocn.2009.01.016, indexed in Pubmed: 19628394.

41. Lehmer LM, Kissel P, Ragsdale BD. Frontal sinus osteoma with osteoblastoma-like histology and associated intracranial pneumatocele. Head Neck Pathol. 2012; 6(3): 384-388, doi: 10.1007/s12105-0120332-0, indexed in Pubmed: 22274656.

42. Kinsley S, Dougherty J. Tension pneumocephalus related to an epidermoid tumor of ethmoid sinus origin. Annals of Emergency Medicine. 1993; 22(2): 259-261, doi: 10.1016/s0196-0644(05)80217-3.

43. Machicado JD, Varghese JM, Orlander PR. Cabergoline-induced pneumocephalus in a medically treated macroprolactinoma. J Clin Endocrinol Metab. 2012; 97(10): 3412-3413, doi: 10.1210/jc.2012-2245, indexed in Pubmed: 22802088.

44. Wang HC, Hwang JC, Peng JP, et al. Tension pneumocephalus-a rare complication of radiotherapy: a case report. J Emerg Med. 2006; 31(4): 387-389, doi: 10.1016/j.jemermed.2006.04.013, indexed in Pubmed: 17046479.

45. Qu X, Yang W, Han T, et al. Pneumocephalus after interventional therapy in esophageal cancer. Clin Neurol Neurosurg. 2010; 112(8): 707-709, doi: 10.1016/j.clineuro.2010.04.013, indexed in Pubmed: 20452718.

46. Swaid SN, Windham TL, Morawetz RB. Pneumocephalus secondary to spontaneous bronchial-subarachnoid fistula. Neurosurgery. 1983; 
13(1): 72-73, doi: 10.1227/00006123-198307000-00016, indexed in Pubmed: 6877571.

47. Holmes M, Martin L, Hanckel R. Spontaneous pneumocephalus secondary to sarcoma of the ethmoid. Ann Otol Rhinol Laryngol. 1957; 66(4): 1021-1028, doi: 10.1177/000348945706600411, indexed in Pubmed: 13498579.

48. lacoangeli M, Di Rienzo A, Fianchini A, et al. Acute tension pneumocephalus secondary to whole spine pneumorrhachis as an unusual presentation of a colon cancer complicated by a transsacral cerebrospinal fluid leak. J Clin Neurosci. 2013; 20(3): 469-471, doi: 10.1016/j.jocn.2012.02.032, indexed in Pubmed: 23164825.

49. Patel MR, Idicula W, Carrau RL, et al. Esophageal-subarachnoid fistula: a case of spontaneous tension pneumocephalus in the setting of esophageal cancer. Head Neck. 2014; 36(6): E52-E56, doi: 10.1002/hed.23459, indexed in Pubmed: 24038386.

50. Ghimire P, Shapey J, Bodi I, et al. Spontaneous tension pneumocephalus and pneumoventricle in ecchordosis physaliphora: case report of a rare presentation and review of the literature. Br J Neurosurg. 2020; 34(5): 537-542, doi: 10.1080/02688697.2019.1594695, indexed in Pubmed: 31079493.

51. Lynn AM, Stuntz JT, Ward BH. Pneumocephalus: an unusual presentation of a persistent neurenteric fistula. J Pediatr. 1978; 93(5): 818820, doi: 10.1016/s0022-3476(78)81091-9, indexed in Pubmed: 712493.

52. Tran P, Reed EJM, Hahn F, et al. Incidence, radiographical features, and proposed mechanism for pneumocephalus from intravenous injection of air. West J Emerg Med. 2010; 11(2): 180-185, indexed in Pubmed: 20823969.

53. Laurent PE, Coulange M, Bartoli C, et al. Retrograde cerebral venous air embolism: a rare cause of intracranial gas. Diagn Interv Imaging. 2014; 95(11): 1113-1115, doi: 10.1016/j.diii.2013.09.009, indexed in Pubmed: 24589189.

54. Syed ON, Weintraub D, DeLaPaz R, et al. Venous air emboli from intravenous catheterization: a report of iatrogenic intravascular pneumocephalus. J Clin Neurosci. 2009; 16(10): 1361-1362, doi: 10.1016/j. jocn.2008.10.024, indexed in Pubmed: 19556135.

55. Işıkay S. Spontaneous Pneumocephalus Secondary to Positive Ventilation in an Infant. Indian J Pediatr. 2019; 86(4): 390-391, doi: 10.1007/s12098-018-2808-8, indexed in Pubmed: 30617608.

56. Park CY, Kim KS. Spontaneous pneumocephalus associated with pneumocele of the frontal sinus. Cephalalgia. 2010; 30(11): 14001402, doi: $10.1177 / 0333102410378047$, indexed in Pubmed: 20663855.

57. Barry C, Rahmani G, Bergin D. Pneumocephalus and Meningitis as Complications of Mastoiditis. Case Rep Radiol. 2019; 2019: 7876494, doi: 10.1155/2019/7876494, indexed in Pubmed: 30915252.

58. Eggerstedt M, Hong S, Eddelman DB, et al. Spontaneous Otogenic Pneumocephalus: Case Series and Update on Management. J Neurol Surg B Skull Base. 2019; 80(4): 424-430, doi: 10.1055/s-00381676036, indexed in Pubmed: 31316888.

59. Harth C, Peeters I, Van Hoe L, et al. Intrathecal gadolinium-enhanced MR-cisternography for the detection of a petrous bone defect in a patient with spontaneous intraventricular pneumocephalus. Acta Neurol Belg. 2020; 120(3): 695-698, doi: 10.1007/s13760-019-01121-7, indexed in Pubmed: 30919289.

60. Pollaers K, Kuthubutheen J. Spontaneous Otogenic Pneumocephalus due to Frequent Plane Travelling. Case Rep Otolaryngol. 2019; 2019: 8768506, doi: 10.1155/2019/8768506, indexed in Pubmed: 31016060 .
61. Wu YH, Yeh IJ, Liu KT. Spontaneous pneumocephalus and subdural hemorrhage after sneezing. Am J Emerg Med. 2018; 36(10): 1928. e1-1928.e3, doi: 10.1016/j.ajem.2018.07.002, indexed in Pubmed: 29980486.

62. Young M, Putty M, Schaible K. Spontaneous Intraventricular Tension Pneumocephalus: Case Report and Review of the Literature. World Neurosurg. 2018; 114: 317-322, doi: 10.1016/j.wneu.2018.03.076, indexed in Pubmed: 29555613.

63. Arai N, Tabuse M, Nakamura A, et al. Spontaneous Intraventricular Pneumocephalus. J Craniofac Surg. 2017; 28(3): 738-740, doi: 10.1097/SCS.0000000000003392, indexed in Pubmed: 28085764.

64. Guleria V, Sharma A, Sharda C, et al. Pneumocephalus presenting as sudden thunderclap headache. Asian Journal of Neurosurgery. 2017; 12(4): 695, doi: 10.4103/ajns.ajns_25_15.

65. Wannemuehler TJ, Hubbell RD, Nelson RF. Tension Pneumocephalus Related to Spontaneous Skull Base Dehiscence in a Patient on BiPAP. Otol Neurotol. 2016; 37(9): e322-e324, doi: 10.1097/ MAO.0000000000001141, indexed in Pubmed: 27636394.

66. Odani N, Kitazono H, Deshpande GA, et al. Severe Sepsis due to Otogenic Pneumococcal Meningitis with Pneumocephalus without Meningeal Symptoms. Intern Med. 2015; 54(13): 1661-1664, doi: 10.2169/internalmedicine.54.4074, indexed in Pubmed: 26134202.

67. Ratre S, Yadav Y, Choudhary S, et al. Pneumocephalus in cerebellopontine angle and meningitis secondary to chronic otitis media in a child. J Neurosci Rural Pract. 2015; 6(3): 413-416, doi: 10.4103/09763147.158795, indexed in Pubmed: 26167031.

68. Ginat DT, Moonis G. Barotrauma-induced Pneumolabyrinth and Pneumocephalus Associated With Semicircular Canal Dehiscence. Otol Neurotol. 2016; 37(4): e176-e177, doi: 10.1097/ MA0.0000000000000628, indexed in Pubmed: 25275873.

69. Pishbin E, Azarfardian N, Salarirad M, et al. Spontaneous Nontraumatic Pneumocephalus: A Case Report. Iran Red Crescent Med J. 2015; 17(7): e23920, doi: 10.5812/ircmj.23920v2, indexed in Pubmed: 26421176.

70. Remenschneider A, Santos F. Pneumocephalus, hearing loss, and vertigo after airline flight in a patient with superior canal dehiscence. In reply. Otol Neurotol. 2015; 36(3): 561-562, doi: 10.1097/ mao.0000000000000493, indexed in Pubmed: 25823355.

71. Rabello FA, Massuda ET, Oliveira JA, et al. Otogenic spontaneous pneumocephalus: case report. Braz J Otorhinolaryngol. 2013; 79(5): 643, doi: 10.5935/1808-8694.20130115, indexed in Pubmed: 24141683.

72. Tamura Y, Suzuki M, Yamamoto M, et al. Extensive pneumocephalus extending into the lateral ventricles from a brain abscess: an intracranial complication of cholesteatoma. J Laryngol Otol. 2013; 127(2): 192-195, doi: 10.1017/S0022215112002848, indexed in Pubmed: 23217411.

73. Javan R, Duszak R, Eisenberg AD, et al. Spontaneous pneumocephalus after commercial air travel complicated by meningitis. Aviat Space Environ Med. 2011; 82(12): 1153-1156, doi: 10.3357/ asem.3100.2011, indexed in Pubmed: 22195398.

74. Mohammed EIR, Profant M. Spontaneous otogenic pneumocephalus. Acta Otolaryngol. 2011; 131(6): 670-674, doi: 10.3109/00016489.2010.541941, indexed in Pubmed: 21254960.

75. Zhao N, Wang DD, Huang $X$, et al. Spontaneous otogenic pneumocephalus presenting with occipital subcutaneous emphysema as primary symptom: could tension gas cause the destruction of cranial bones? J Neurosurg. 2011; 115(4): 679-683, doi: 10.3171/2011.6.JNS11104, indexed in Pubmed: 21740114. 
76. Lee JS, Park YS, Kwon JT, et al. Spontaneous pneumocephalus associated with pneumosinus dilatans. J Korean Neurosurg Soc. 2010; 47(5): 395-398, doi: 10.3340/jkns.2010.47.5.395, indexed in Pubmed: 20539803.

77. Murugesan C, Powell M, Khayal HB. Sinus barotrauma leading to extradural muco-pneumocephalus. Br J Neurosurg. 2010; 24(1): 80-81, doi: 10.3109/02688690903506069, indexed in Pubmed: 20158359.

78. Abbati SG, Torino RR. Spontaneous intraparenchymal otogenic pneumocephalus: A case report and review of literature. Surg Neurol Int. 2012; 3: 32, doi: 10.4103/2152-7806.93861, indexed in Pubmed: 22530167.

79. Pennings RJE, Liauw L, Cremers CW. A spontaneous otogenic extradural pneumocephalus. Otol Neurotol. 2009; 30(6): 864, doi: 10.1097/ MA0.0b013e31818de597, indexed in Pubmed: 18931642.

80. Hyam JA, Morgan L, Mendoza ND. Coma caused by spontaneous otogenic pneumocephalus. Clin Neurol Neurosurg. 2008; 110(1): 62-64, doi: 10.1016/j.clineuro.2007.08.008, indexed in Pubmed: 17942218.

81. Mathai J, Ahammed S, Pushpakumari KP, et al. Pneumocephalus presenting as a complication of chronic otitis media - a case report. Indian J Otolaryngol Head Neck Surg. 2008; 60(4): 390-392, doi: 10.1007/s12070-008-0124-8, indexed in Pubmed: 23120591.

82. Bhattacharyya N, Friedlander RM. Images in clinical medicine. Pneumocephalus associated with pneumosinus dilatans frontalis. N Engl J Med. 2007; 357(11): 1136, doi: 10.1056/NEJMicm062822, indexed in Pubmed: 17855674.

83. Ciorba A, Berto A, Borgonzoni M, et al. Pneumocephalus and meningitis as a complication of acute otitis media: case report. Acta Otorhinolaryngol Ital. 2007; 27(2): 87-89, indexed in Pubmed: 17608137.

84. Jensen MB, Adams HP. Pneumocephalus after air travel. Neurology. 2004; 63(2): 400-401, doi: 10.1212/01.wnl.0000130264.66211.74, indexed in Pubmed: 15277656.

85. Schrijver HM, Berendse HW. Pneumocephalus by Valsalva's maneuver. Neurology. 2003; 60(2): 345-346, doi: 10.1212/01. wnl.0000033802.04869.14, indexed in Pubmed: 12552062.

86. Añorbe E, Aisa P, Saenz de Ormijana J. Spontaneous pneumatocele and pneumocephalus associated with mastoid hyperpneumatization. Eur J Radiol. 2000; 36(3): 158-160, doi: 10.1016/s0720-048x(00)00177-7, indexed in Pubmed: 11091017.

87. Babl FE, Arnett AM, Barnett E, et al. Atraumatic pneumocephalus: a case report and review of the literature. Pediatr Emerg Care. 1999; 15(2): 106-109, indexed in Pubmed: 10220080.

88. Boninsegna $\mathrm{E}$, Barillari M, De Rossi S, et al. Non-traumatic pneumocephalus caused by increased depth of olfactory fossa. $\mathrm{Br}$ J Oral Maxillofac Surg. 2019; 57(6): 587-589, doi: 10.1016/j. bjoms.2019.01.025, indexed in Pubmed: 31085015.

89. Gaberel T, Ponte KF, Khouri S, et al. Arachnoid cyst associated to spontaneous CSF fistula and massive pneumocephalus. Acta Neurochir (Wien). 2012; 154(10): 1941-1942, doi: 10.1007/s00701-0121465-3, indexed in Pubmed: 22878454.

90. Nash R, Wilson M, Adams M, et al. Spontaneous pneumocephalus presenting with alien limb phenomena. J Laryngol Otol. 2012; 126(7): 733-736, doi: 10.1017/S002221511200093X, indexed in Pubmed: 22642786.

91. Hogg RP, Pillay H, Mitchell R, et al. Idiopathic CSF rhinorrhoea presenting with tension pneumocephalus and hemiparesis. J Laryngol Otol. 1998; 112(7): 654-656, doi: 10.1017/s0022215100141362, indexed in Pubmed: 9775298.
92. Mirzai S, Rifai AO, Webb S, et al. Probable pneumocephalus in a user of natural remedies with newly diagnosed diabetes mellitus type 1 . IDCases. 2019; 17: e00581, doi: 10.1016/j.idcr.2019.e00581, indexed in Pubmed: 31312601.

93. Saleem S, Anwar A, Aslam H, et al. Non-Traumatic Pneumocephalus and Sub-Dural Empyema as a Complication of Chronic Sinusitis. Cureus. 2019; 11(7): e5202, doi: 10.7759/cureus.5202, indexed in Pubmed: 31565608.

94. Sun X, Chiang GC. Pneumocephalus as a Rare Presentation of Acute Sinusitis with Intracranial Extension - Two Cases and a Review of Literature. J Clin Imaging Sci. 2019; 9: 26, doi: 10.25259/JCIS-26-2019, indexed in Pubmed: 31448177.

95. Ansari AS, Dennis BB, Shah D, et al. An unusual case of infective pneumocephalus: case report of pneumocephalus exacerbated by continuous positive airway pressure. BMC Emerg Med. 2018; 18(1): 2, doi: 10.1186/s12873-018-0154-9, indexed in Pubmed: 29347913.

96. Kumari A, Agrawal SC. Neonatal meningitis complicating with pneumocephalus. J Neurosci Rural Pract. 2014; 5(1): 68-70, doi: 10.4103/0976-3147.127879, indexed in Pubmed: 24741257.

97. Srikumar T, Pabbathi S, Fernandez J, et al. Aspergillus Terreus Brain Abscess Complicated by Tension Pneumocephalus in a Patient with Angiosarcoma. Am J Case Rep. 2017; 18: 33-37, doi: 10.12659/ ajcr.900425, indexed in Pubmed: 28070116.

98. Lin K. Pneumocephalus in an advanced aged woman. Chinese Medical Journal. 2014; 127(23): 4157-4158.

99. Ramakrishnan S, Krishnan P, Chandra P, et al. Pneumocephalus in mixed aerobic and anaerobic (Bacteroides fragilis) meningitis. Indian Journal of Pathology and Microbiology. 2014; 57(1): 160, doi: 10.4103/0377-4929.130940.

100. Kosac Vd, Matta APC, Prado FM, et al. Tension pneumocephalus and rhinorrhea related to chronic sinusitis. Arq Neuropsiquiatr. 2013; 71(4): 269, doi: 10.1590/0004-282x20130017, indexed in Pubmed: 23588294.

101. Rota E, Sacchini D, Paolillo F, et al. Pneumocephalus as uncommon presentation of pneumococcal meningitis. Neurol India. 2013; 61(3): 314315, doi: 10.4103/0028-3886.115080, indexed in Pubmed: 23860160.

102. Kaur S, Seth A, Narula MK. Pneumocephalus: a rare complication of meningitis. Indian J Pediatr. 2012; 79(11): 1537-1538, doi: 10.1007/s12098-012-0774-0, indexed in Pubmed: 22576296.

103.Bhogal P, Bhatnagar G, Manieson J, et al. An unusual case of pneumocephalus. BMJ Case Rep. 2011; 2011, doi: 10.1136/ bcr.06.2010.3106, indexed in Pubmed: 22673713.

104. Kumari A, Agrawal SC. Pneumocephalus consequent to staphylococcal pneumonia and meningitis. J Pediatr Neurosci. 2011; 6(1): 8486, doi: 10.4103/1817-1745.84420, indexed in Pubmed: 21977100.

105. Oh KP, Lee YJ, Bae SC, et al. Intractable progressive pneumocephalus due to tuberculous meningoencephalitis. Neurology. 2011; 77(6): 600-601, doi: 10.1212/WNL.0b013e318228c125, indexed in Pubmed: 21825275.

106. Damergis JA, Chee K, Amitai A. Otogenic pneumococcal meningitis with pneumocephalus. J Emerg Med. 2010; 39(3): e109-e112, doi: 10.1016/j.jemermed.2007.10.082, indexed in Pubmed: 18597973.

107. Aghaei Lasboo A, Walker MT, Hijaz TA. An unusual appearance of discitis due to gas-forming Escherichia coli with associated pneumocephalus. Spine (Phila Pa 1976). 2010; 35(7): E257-E259, doi: 10.1097/BRS.0b013e3181bca12f, indexed in Pubmed: 20228705.

108. Chung SJ, Wan-Teck L, Tham SC, et al. Osteoradionecrosis of the cervical spine complicated by pneumocephalus and meningitis in a nasopharyngeal cancer patient radically treated with radiotherapy 11 years ago. 
BMJ Case Rep. 2009; 2009, doi: 10.1136/bcr.11.2008.1179, indexed in Pubmed: 21686429.

109. Hama-Amin AD, Gilhuis HJ, Portegies P. Teaching neuroimages: pneumocephalus due to bacterial meningitis. Neurology. 2009; 73(10): e53, doi: 10.1212/WNL.0b013e3181b6bbcf, indexed in Pubmed: 19738168.

110. Sreejith P, Vishad V, Pappachan JM, et al. Pneumocephalus as a complication of multidrug-resistant Klebsiella pneumoniae meningitis. Eur J Intern Med. 2008; 19(2): 140-142, doi: 10.1016/j.ejim.2007.03.021, indexed in Pubmed: 18249312.

111. Townend BS, Whyte S, Sturm JW. Cerebral abscess with pneumocephalus. Intern Med J. 2005; 35(7): 429, doi: 10.1111/j.1445-5994.2005.00854.x, indexed in Pubmed: 15958115.

112. Parmar MS. Pneumocephalus associated with Bacteroides fragilis meningitis. J Postgrad Med. 2004; 50(4): 272-273, indexed in Pubmed: 15623969.

113. Pooboni SK, Mathur SK, Dux A, et al. Pneumocephalus in neonatal meningitis: diffuse, necrotizing meningo-encephalitis in Citrobacter meningitis presenting with pneumatosis oculi and pneumocephalus. Pediatr Crit Care Med. 2004; 5(4): 393-395, doi: 10.1097/01. pcc.0000129136.45258.af, indexed in Pubmed: 15215013.

114. Sedaghatian MR, Ramachandran P, Rashid N. Diffuse pneumocephalus caused by neonatal Enterobacter cloacae meningitis. Arch Dis Child Fetal Neonatal Ed. 2004; 89(4): F324, doi: 10.1136/adc.2003.033209, indexed in Pubmed: 15210666.

115. Kassim Z, Aziz AA, Haque QM, et al. Isolation of Proteus mirabilis from severe neonatal sepsis and central nervous system infection with extensive pneumocephalus. Eur J Pediatr. 2003; 162(9): 644-645, doi: 10.1007/s00431-003-1240-9, indexed in Pubmed: 12836017.

116. Goyal M, Sharma R, Berry M. Diffuse pneumocephalus due to meningitis: CT findings. Pediatr Radiol. 1996; 26(4): 278-279, doi: 10.1007/ BF01372112, indexed in Pubmed: 8677146.

117. Randall JM, Hall K, Coulthard MG. Diffuse pneumocephalus due to Clostridium septicum cerebritis in haemolytic uraemic syndrome: CT demonstration. Neuroradiology. 1993; 35(3): 218-220, doi: 10.1007/ BF00588500, indexed in Pubmed: 8459926.

118. Finelli PF, Bergen R. Spontaneous pneumocephalus with meningitis: CT demonstration. J Comput Assist Tomogr. 1991; 15(3): 525-526, doi: 10.1097/00004728-199105000-00041, indexed in Pubmed: 2026827.

119. Holtby I. Comment on Otogenic brain abscess with pneumocephalus. Infection. 1991; 18(1): 8000.

120. Klein MA, Kelly JK, Jacobs IG. Diffuse pneumocephalus from Clostridium perfringens meningitis: CT findings. AJNR Am J Neuroradiol. 1989; 10(2): 447, indexed in Pubmed: 2539004.

121. Iplikcioglu AC, Karabag H. Frontoethmoid Osteoma Causing Tension Pneumocephalus. J Neurosci Rural Pract. 2019; 10(3): 548-550, doi: 10.1055/s-0039-1698292, indexed in Pubmed: 31595133.

122. Elabd SS, Ahmad MM, Qetab SQ, et al. Cabergoline-Induced Pneumocephalus Following Treatment for Giant Invasive Macroprolactinoma Presenting With Spontaneous Cerebrospinal Fluid Rhinorrhea. Clin Med Insights Endocrinol Diabetes. 2018; 11: 1179551418758640, doi: 10.1177/1179551418758640, indexed in Pubmed: 29467589.

123. AlbertA, Allbright R, Nichols T, etal. Pneumocephalus after the Treatment of an Inoperable Superior Sulcus Tumor with Chemoradiation. Case Rep Oncol Med. 2017; 2017: 3016517, doi: 10.1155/2017/3016517, indexed in Pubmed: 29318068.

124. Hackenbroch C, Kleinagel U, Hossfeld B. Tension Pneumocephalus Due to an Osteoma of the Frontal Sinus. Dtsch Arztebl Int. 2017; 114(31-32): 534, doi: 10.3238/arztebl.2017.0534, indexed in Pubmed: 28835327.

125. Umredkar $A B$, Disawal $A$, Anand $A$, et al. Frontal sinus osteoma with pneumocephalus: A rare cause of progressive hemiparesis. Indian J Ra- diol Imaging. 2017; 27(1): 46-48, doi: 10.4103/0971-3026.202972, indexed in Pubmed: 28515584.

126. Jimenez-Jimenez E, Martí SS, Villas MV. Tension pneumocephalus related to radiotherapy for nasopharyngeal carcinoma. Case Rep Oncol Med. 2014; 2014: 327380, doi: 10.1155/2014/327380, indexed in Pubmed: 25210637.

127. Marchant B, Sheele JM. A novel cause of pneumocephalus. J Emerg Med. 2013; 44(6): e381-e383, doi: 10.1016/j.jemermed.2012.11.070, indexed in Pubmed: 23453428.

128. Nanba K, Usui T, Nakakuki T, et al. Tension pneumocephalus after administration of two $0.25 \mathrm{mg}$ cabergoline tablets in MEN1-related macroprolactinoma. BMJ Case Rep. 2013; 2013, doi: 10.1136/bcr2013-009986, indexed in Pubmed: 23749864.

129. Guedes Bd, da Rocha AJ, da Silva CJ, et al. A rare association of tension pneumocephalus and a large frontoethmoidal osteoma: imaging features and surgical treatment. J Craniofac Surg. 2011; 22(1): 212-213, doi: 10.1097/SCS.0b013e3181f76031, indexed in Pubmed: 21233751.

130. Chung SJ, Wan-Teck L, Tham SC, et al. Osteoradionecrosis of the cervical spine complicated by pneumocephalus and meningitis in a nasopharyngeal cancer patient radically treated with radiotherapy 11 years ago. BMJ Case Rep. 2009; 2009, doi: 10.1136/bcr.11.2008.1179, indexed in Pubmed: 21686429.

131. Torres A, Holoye PY, Camacho LH. Spontaneous pneumocephalus associated with recurrent colorectal carcinoma. J Clin Oncol. 2008; 26(33): 5483-5484, doi: 10.1200/JC0.2008.19.4381, indexed in Pubmed: 18955437.

132. Bramley DC, Ghosh S. Tension pneumocephalus attributable to an ethmoid osteoma presenting as a stroke in evolution: an unusual presentation. Emerg Med J. 2001; 18(4): 317-318, doi: 10.1136/emj.18.4.317, indexed in Pubmed: 11435382.

133. Wu CT, Lee ST. Delayed spontaneous tension pneumocephalus caused by radionecrosis of the skull base. Br J Neurosurg. 1999; 13(2): 214-216, doi: 10.1080/02688699944032, indexed in Pubmed: 10616596.

134. Jakubowski E, Kirsch E, Mindermann T, et al. Intradiploic epidermoid cyst of the frontal bone presenting with tension pneumocephalus. Acta Neurochir (Wien). 1997; 139(1): 86-87, doi: 10.1007/BF01850874, indexed in Pubmed: 9059718.

135. Kiu MC, Wan YL, Ng SH, et al. Pneumocephalus due to nasopharyngeal carcinoma: case report. Neuroradiology. 1996; 38(1): 70-72, doi: 10.1007/BF00593227, indexed in Pubmed: 8773283.

136. Ng WF, Fung KH, Sham JS. Tension pneumocephalus-a rare complication of radiotherapy in nasopharyngeal carcinoma. Pathology. 1995; 27(2): 204-208, doi: 10.1080/00313029500169902, indexed in Pubmed: 7567156.

137. Banerjee T, Meagher JN, Donley C. Osteoid osteoma of the ethmoid and pneumocephalus. South Med J. 1975; 68(1): 90-93, doi: 10.1097/00007611-197501000-00024, indexed in Pubmed: 1154067.

138. Wilson ES, Sheft DJ. Epidermoid tumor of the skull with intracranial pneumatocele. Case report. J Neurosurg. 1968; 28(6): 600-602, doi: 10.3171/jns.1968.28.6.0600, indexed in Pubmed: 5671297.

139. Akashi K, Saegusa J, Morinobu A. Pneumocephalus in granulomatosis with polyangiitis. Rheumatology (Oxford). 2016; 55(1): 108, doi: 10.1093/rheumatology/kev362, indexed in Pubmed: 26385371.

140. Miyaji Y, Kurokawa T, Tanaka F, et al. Meningitis with pneumocephalus originating from a sacral pressure ulcer. Intern Med. 2013; 52(18): 2163-2164, doi: 10.2169/internalmedicine.52.0935, indexed in Pubmed: 24042536. 